Chapter 2

\title{
Antennas for Body Centric Wireless Communications at Millimeter Wave Frequencies
}

\author{
Nacer Chahat, Maxim Zhadobov and \\ Ronan Sauleau \\ Additional information is available at the end of the chapter \\ http://dx.doi.org/10.5772/58816
}

\section{Introduction}

Over the past few years, wearable antennas for body centric wireless communication systems have been increasingly gaining attention. Body area networks (BANs) are wireless communication systems that enable communications between wearable and/or implanted electronic devices.

Such systems are of great interest for various applications such as healthcare, entertainment, military, identification systems, sport, smart home, and space [1, 2]. Indeed, portable devices, incorporating antennas close to the human body, have been used for many decades by the military. Nowadays, in order to increase overall effectiveness of soldiers on the battlefield, works are undertaken to integrate wireless systems to all equipment, such as weapons, sighting systems, helmet, and so on. Besides, wearable or implanted sensors increase the ability of doctors to monitor their patients at long distance and in real time. This monitoring capability is also used in sport applications and in rescue worker interventions.

Recently, studies carried out at millimeter waves have grown rapidly. This is due to the fact that many advantages can be found in operating such systems at millimeter waves compared to microwaves. First, because of the large available spectrum (7 GHz worldwide), very high data rates can be reached (up to $5 \mathrm{~Gb} / \mathrm{s}$ ) [3]. Second, it provides a high level of security and low interference with adjacent networks [4]. Finally, compared to on-body devices operating at microwaves, the size of similar millimeter-wave systems is significantly reduced.

Hence, the use of millimeter wave systems for BANs will have a high impact, in particular in the defense sector (Fig. 1), where communications emanating from a dismounted soldier leads to detection, location and vulnerability to enemy attack. The high atmospheric attenuation in 
the 60-GHz band will lead to much higher levels of security against detection, interception and jamming. Fig. 1 illustrates a scenario of soldier-to-soldier communications for covert battlefield operation where co-located soldiers are wirelessly networked to allow high-speed communications within a cluttered urban warfare environment. Besides, every soldier is equipped with advanced technology significantly improving situational awareness, lethality and survivability such as GPS, helmet mounted display, RADAR bullet detector, etc.

In addition, millimeter wave BANs will also benefit civilian sectors such as healthcare, personal entertainment, sports training, and emergency services. In hospital, clinics, entertainment venues, and public transport, there is a need to relay personalized data to and from individuals, in confined areas, or in crowds, and the high frequency and highly directive beams from small millimeter-wave antennas will reduce interference between users and other communication equipment.

The aim of this book chapter is to provide a review of recent progresses and outstanding challenges in the field of antennas for body-centric communication at millimeter waves.

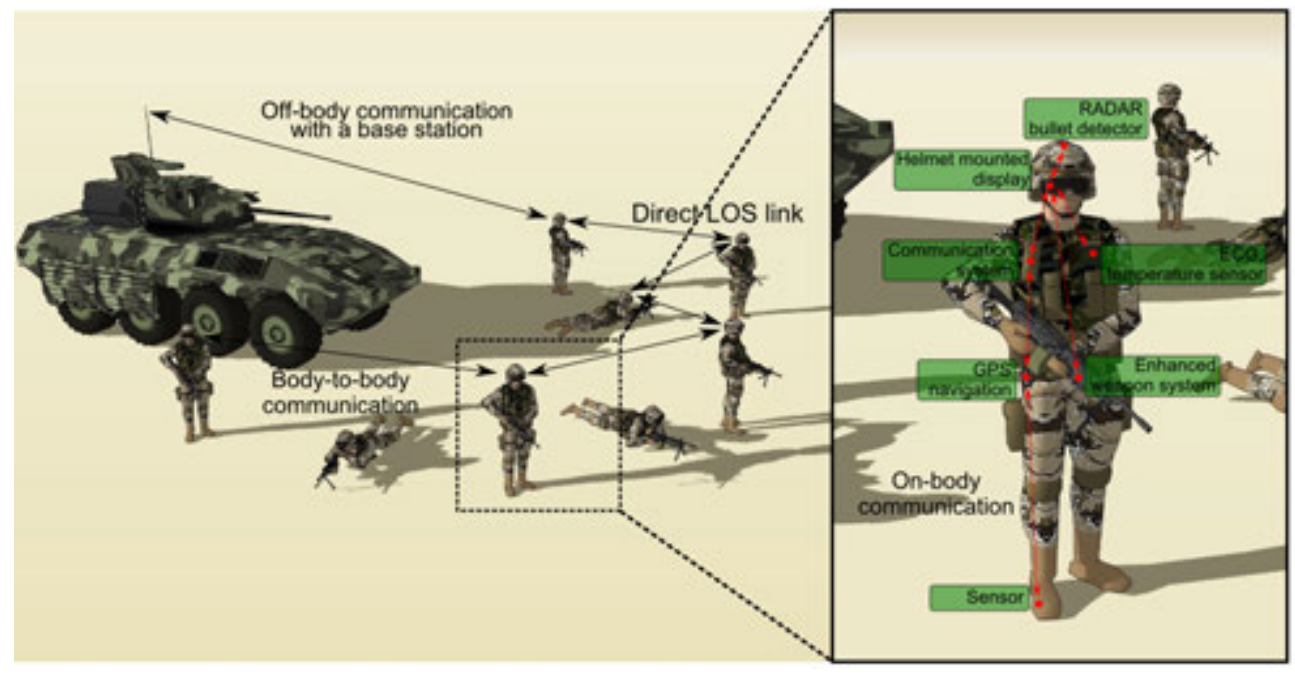

Figure 1. Soldier-to-soldier communications for covert battlefield operations. The black arrows represent some possible wireless links allowing data transfer from one soldier to another.

\section{Electromagnetic properties and modeling of the human body}

In this Section, the electromagnetic properties and modeling of the human body are investigated. First, to study the interaction of millimeter waves and the human body, the skin dielectric properties are carefully characterized. Then, the influence of the antenna feeding is investigated. Then, as the dielectric properties of the skin have been assessed, a numerical 
model of the human body is introduced using a Debye model. Finally, a semi-solid phantom is introduced for antenna measurement in close proximity to the body.

\subsection{Interaction of millimeter waves with the human body}

The primary biological targets of 60-GHz radiations are the skin and eyes. Exposure of the eyes leads to the absorption of the EM energy by the cornea characterized by a free water content of $75 \%$ and a thickness of $0.5 \mathrm{~mm}$. Ocular lesions have been found after high-intensity exposure of the eye $\left(3 \mathrm{~W} / \mathrm{cm}^{2}, 6 \mathrm{~min}\right)$ [5]. However, studies performed at $60 \mathrm{GHz}\left(10 \mathrm{~mW} / \mathrm{cm}^{2}, 8 \mathrm{~h}\right)$ demonstrated no detectable physiological modifications [6], indicating that millimeter waves act on the cornea in a dose-dependent manner.

Hereafter we will essentially consider the interactions with the skin as it covers $95 \%$ of the human body surface. From the EM viewpoint, human skin can be considered as an anisotropic multilayer dispersive structure made of three different layers, namely, epidermis, dermis, and subcutaneous fat layer (Fig. 2). The skin also contains capillaries and nerve endings. It is mainly composed of $65.3 \%$ of free water, $24.6 \%$ of proteins, and $9.4 \%$ of lipids [7].

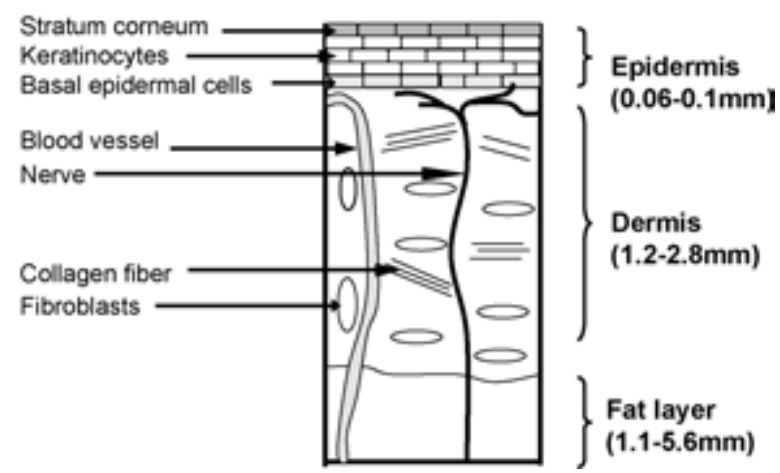

Figure 2. Schematic representation of the skin structure.

Knowledge of the dielectric properties of the skin is essential for the determination of the reflection from, transmission through, and absorption in the body, as well as for EM modeling. In contrast to frequencies below $20 \mathrm{GHz}$, existing data on the permittivity of tissues in the millimeter-wave band are very limited [8]-[11] due to some technical difficulties. In the 10-100 $\mathrm{GHz}$ range, the dispersive dielectric properties of the skin and biological solutions are primarily related to the rotational dispersion of free water molecules. In particular, high losses are related to the free water relaxation with the peak at $23 \mathrm{GHz}$ at $33^{\circ} \mathrm{C}$.

In contrast to frequencies below $20 \mathrm{GHz}$, the already-existing data on the relative permittivity of human tissues at millimeter waves are very limited. In addition, the results reported so far in the literature strongly depend on the measurement technique, the sample type (in vivo or in vitro study) and other experimental conditions such as skin temperature, location on the body and thickness of different skin layers. 
Table 1 provides a summary of the data previously reported at $60 \mathrm{GHz}$. These results show that the literature data vary significantly from one study to another depending on the sample type. Besides, since the skin consists of approximately $65 \%$ of free water [7], its complex permittivity is strongly dispersive and temperature-dependent; this should be also taken into account for definition of an accurate skin permittivity model.

To validate our measurement technique and obtain reference data for the skin-equivalent phantom, we performed a measurement campaign on a group of volunteers using two different techniques: open-ended coaxial slim probe [12] and a new method based on heating kinetics [13]. A very good agreement is demonstrated between our measurements and Gabriel [10] and Alekseev [11] data as shown in Fig. 3.

\begin{tabular}{ccccc}
\hline Reference & Complex permittivity $\boldsymbol{\varepsilon}^{*}$ & $\mathbf{T},{ }^{\circ} \mathbf{C}$ & Method & Sample type \\
\hline Gandhi et al. [8] & $8.89-j 13.15$ & $37 \pm 0.5$ & $\mathrm{E}$ & In vitro \\
\hline Alabaster et al. [9] & $9.9-j 9.0$ & 23 & $\mathrm{M}$ vitro \\
\hline $\begin{array}{c}\text { Gabriel et al. [10] } \\
\text { "wet skin" }\end{array}$ & $10.22-j 11.84$ & 37 & $\mathrm{E}$ & In vitro \\
\hline $\begin{array}{c}\text { Gabriel et al.[10] } \\
\text { "dry skin" }\end{array}$ & $7.98-j 10.90$ & $32.5 \pm 0.5$ & $\mathrm{E}$ Ino \\
\hline Alekseev et al. [11] & $8.12-j 11.14$ & $32.5 \pm 0.3$ & $\mathrm{M}$ & In vivo \\
\hline Chahat et al. [12] & $8.02-j 10.5$ & $32.5 \pm 0.5$ & $\mathrm{M}$ In vivo \\
\hline Chahat et al. [13] & $8.4-j 10.96$ & $32.5 \pm 0.5$ & $\mathrm{M}$ & In vivo \\
\hline
\end{tabular}

$\mathrm{E}=$ Extrapolation. $\mathrm{M}=$ Measurement. T=theoretical value.

Table 1. Overview of the skin dielectric properties at $60 \mathrm{GHz}$.

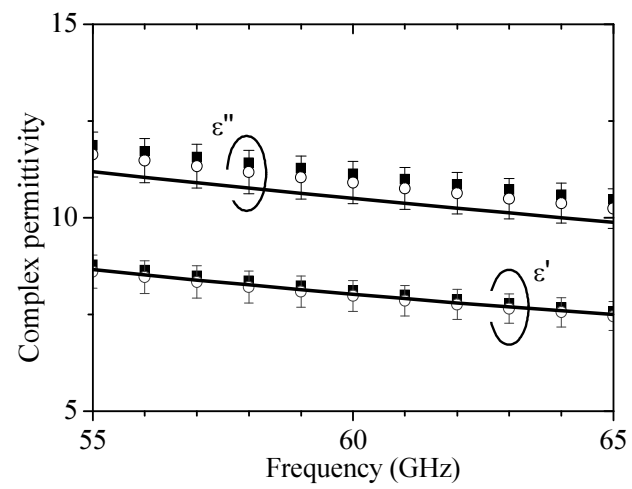

Fig. 3. Comparison of our experimental result for the wrist skin

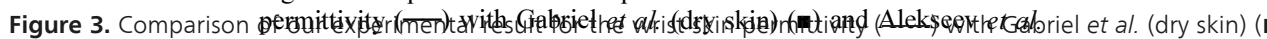

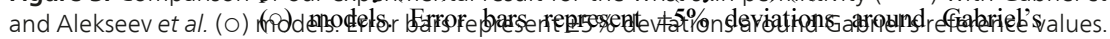
reference values.

\subsection{Numerical skin-equivalent phantom}

Taking into account the very shallow penetration of millimeter waves into the skin (typically $0.5 \mathrm{~mm}$ at $60 \mathrm{GHz}$ ), using homogeneous skin-equivalent phantoms provides accurate results for the antenna / human body interaction evaluation as well as for the propagation channel characterization [28]. For the broadband analysis, dispersive models can be used. Debye model with a single relaxation time $\tau$ equal to that of free water at the same temperature was demonstrated to provide a good accuracy for modeling the experimental permittivity data in the considered frequency range [12]: 


\subsection{Numerical skin-equivalent phantom}

Taking into account the very shallow penetration of millimeter waves into the skin (typically $0.5 \mathrm{~mm}$ at $60 \mathrm{GHz}$ ), using homogeneous skin-equivalent phantoms provides accurate results for the antenna / human body interaction evaluation as well as for the propagation channel characterization [28]. For the broadband analysis, dispersive models can be used. Debye model with a single relaxation time $\tau$ equal to that of free water at the same temperature was demonstrated to provide a good accuracy for modeling the experimental permittivity data in the considered frequency range [12]:

$$
\varepsilon^{*}=\varepsilon_{\infty}+\frac{\Delta \varepsilon}{1+j \omega \tau}+\frac{\sigma}{j \omega \varepsilon_{0}} .
$$

In this equation, $\omega=2 \pi f, f[\mathrm{~Hz}]$ is the frequency, $\Delta \varepsilon=\varepsilon_{s}-\varepsilon_{\infty}$ is the magnitude of the dispersion of the free water fraction of skin, $\varepsilon_{s}$ is the permittivity at $\omega \tau<<1, \varepsilon_{\infty}$ is the optical permittivity, $\varepsilon_{0}=8.8510^{-12} \mathrm{~F} / \mathrm{m}$, and $\sigma[\mathrm{S} / \mathrm{m}]$ is the ionic conductivity. The optimized parameters that fit to the measured permittivity in the 55-65 GHz range are the following: $\varepsilon_{\infty}=4.1, \varepsilon_{s}=34.8, \tau=6.9 \times 10^{-12} \mathrm{~s}$, and $\sigma=0.7 \mathrm{~S} / \mathrm{m}$ [12]. This model allows an accurate representation of typical broadband dielectric properties of dry skin in the numerical modeling.

\subsection{Experimental skin-equivalent phantom}

\subsubsection{Composition}

The main components employed for the fabrication of a homogeneous semi-solid skinequivalent phantom are the following:

- Deionized water. Water is the main constituent of the phantom because it is also the main skin component. It primarily determines the dispersive behavior of the phantom.

- Agar. It is employed for the retention of self-shaping, and its contribution to the phantom dielectric properties is negligible for small concentrations (typically below $4 \%$ ).

- Polyethylene powder. It is used to tune the real and imaginary parts of the phantom permittivity.

- TX-151. Since the agar and polyethylene powder cannot be mixed directly, the viscosity is increased using TX-151.

- Sodium azide (NaN3). It serves as a preservative.

\subsubsection{Fabrication procedure}

The fabrication steps are the following. Deionized water, sodium azide, and agar are mixed in a kettle and heated on a stove, while the mixture is continuously stirred. When this liquid starts boiling, heating is stopped. TX-151 is sprinkled into the liquid and quickly mixed. Then the polyethylene powder is added into the stirred liquid. Finally, the obtained mixture is poured 
into a mold and cooled in the same container for a few hours to room temperature for solidification. Using alginate gel powder, molds with realistic body-specific shapes can be manufactured for the phantom fabrication as illustrated in Fig. 4.

Particular attention should be paid to the following critical points. First, to avoid variations of dielectric properties from one phantom to another, the room temperature should remain identical (in our case $20 \pm 1^{\circ} \mathrm{C}$ ) during the fabrication and further measurements. Second, the type of polyethylene powder is important; we recommend using particles with an average diameter of $20 \mu \mathrm{m}$ and low density $\sim 900-1100 \mathrm{~kg} / \mathrm{m}^{3}$. Finally, to preserve the dielectric properties of the phantom over time, it is important to avoid water evaporation since this would result in a decrease of the permittivity. This can be for instance achieved by wrapping the phantom in a plastic film. More details regarding the phantom preparation procedure can be found in [12].

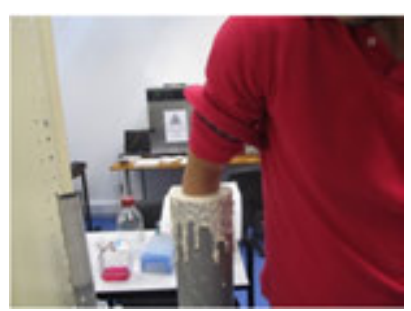

(a)

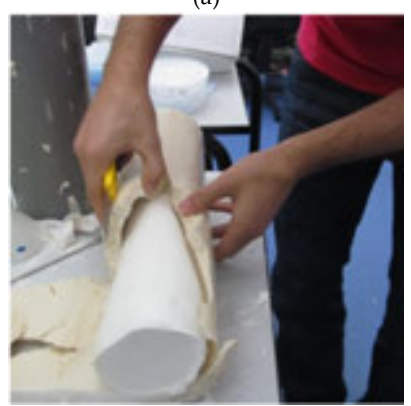

(d)

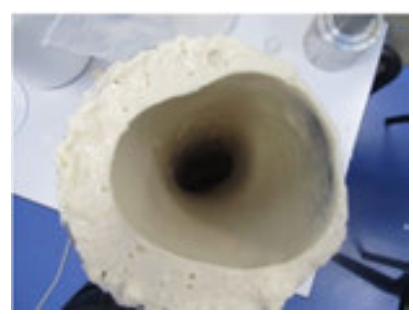

(b)

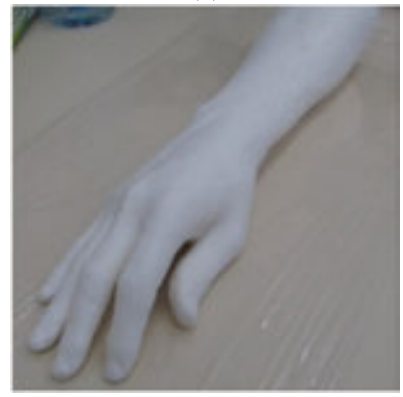

(e)

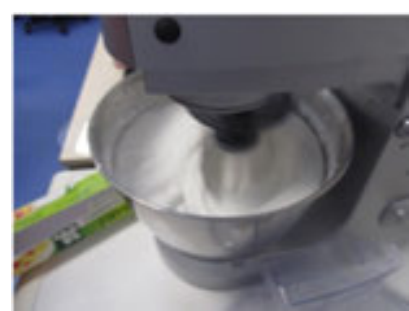

(c)

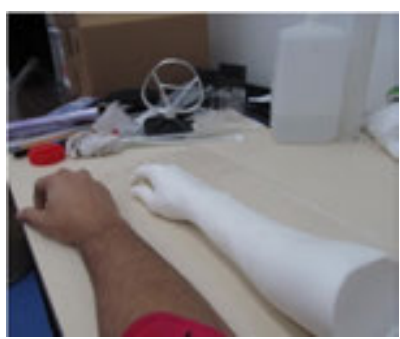

Figure 4. Skin-equivalent phantom representing an arm and a hand: (a) fabrication of an alginate mold; (b) alginate mold of a human

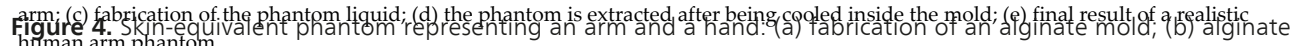
mold of a human arm; (c) fabrication of the phantom liquid; (d) the phantom is extracted after being cooled inside the mold; (e) final result of a realistic human arm phantom.

\subsubsection{Dielectric properties}

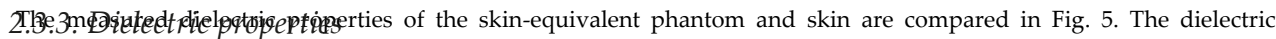
properties of the proposed phantom are within $\pm 10 \%$ of the measured skin permittivity. Table 2 compares the dielectric properties of the phantom measured using the cpaxial probe and the heating kinetics technique [13] to those of the The measured dielectric properties of the SkIn-eguivalent phantom and skin are compared in

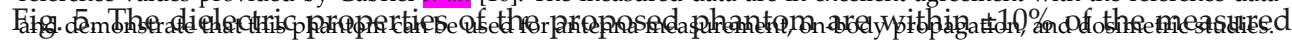
skin permittivity. Table 2 compares the dielectric properties of the phantom measured using the coaxiat probenand the heating kinetics technique [13] to those of the reference values

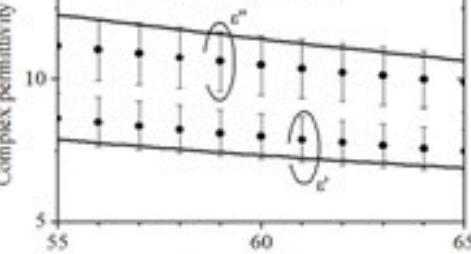


provided by Gabriel et al. [10]. The measured data are in excellent agreement with the reference data and demonstrate that this phantom can be used for antenna measurement, on-body propagation, and dosimetric studies.

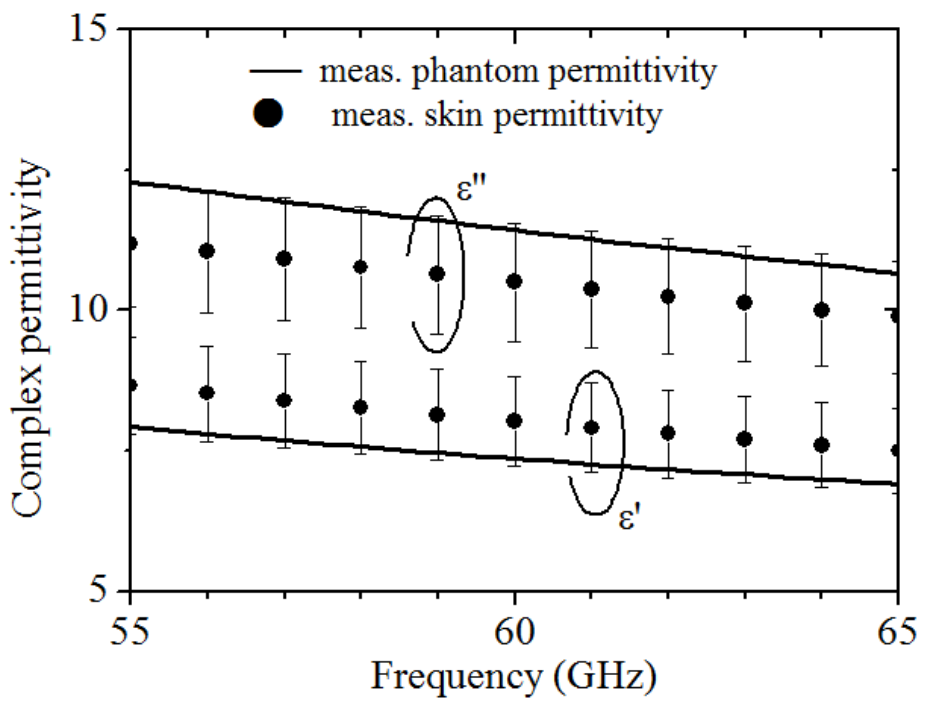

Figure 5. Dielectric properties of the skin-equivalent phantom compared to those of skin [12]. Error bars represent $\pm 10 \%$ of the measured skin permittivity.

\begin{tabular}{ccccc}
\hline & $\boldsymbol{\varepsilon}^{*}$ & $\boldsymbol{\Delta} \boldsymbol{\varepsilon}^{*}$ & $\boldsymbol{R}$ & $\boldsymbol{\delta}(\mathbf{m m})$ \\
\hline $\begin{array}{c}\text { Reference value (Gabriel et al.) } \\
{[10]}\end{array}$ & $7.98-j 10.9$ & - & 0.38 & 0.48 \\
\hline Phantom (coaxial probe) & $7.4-j 11.4$ & $7.3 \%-j 4.6 \%$ & 0.39 & 0.45 \\
\hline Phantom (heating kinetics) & $8.3-j 10.8$ & $4 \%-j 0.9 \%$ & 0.38 & 0.49 \\
\hline
\end{tabular}

Table 2. The dielectric properties of the proposed phantom (using two different techniques) compared to those of the reference data provided by Gabriel et al. [10]. $\Delta \varepsilon^{*}$ is the error relative to Gabriel et al. data.

\subsubsection{Validation}

To further confirm the reliability of this phantom, we performed SAR measurement using a high-performance thermal imaging camera (FLIR SC500, FLIR Systems, Wilsonville, OR, USA) and the measurement set-up shown in Fig. 6a. The SAR assessment methodology is described in Fig. 6b. The temperature dynamic, recorded using the IR camera, is fitted to the onedimensional bio-heat transfer equation [12]. The fitting procedure is performed by minimizing the standard deviation value varying the incident power density (IPD). Once the IPD value 
has been determined, the SAR can be found (Fig. 6b). The simulated and measured SAR results are in excellent agreement (Fig. 6) which confirms the accuracy of this phantom.

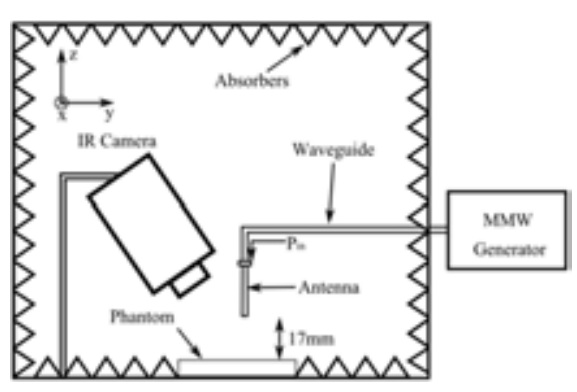

(a)
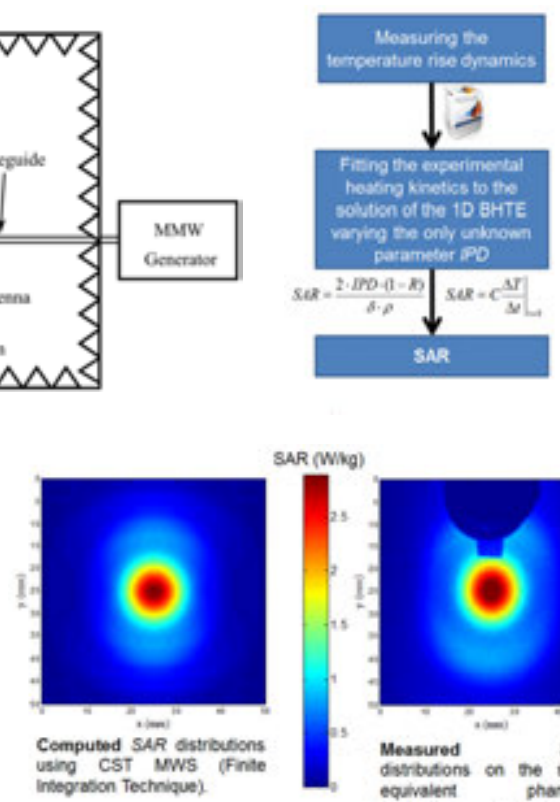

(c)

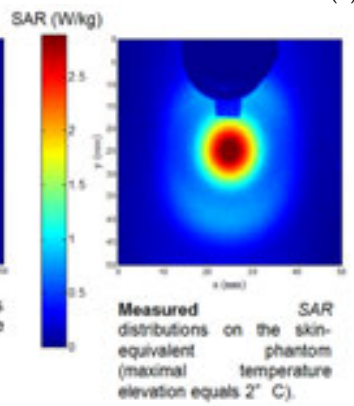

eimation equats $2^{*}$ C)

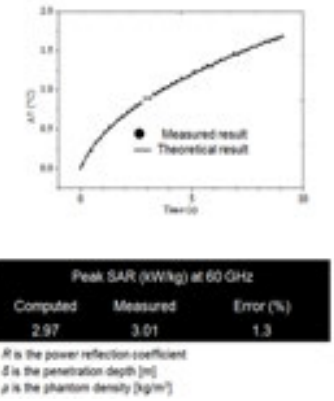

(b)

Figure 6. (a) SAR measurement set-up. (b) SAR assessment methodology from the temperature rise. (c) Simulated and measured SAR

Figure 6. (a) SAR measurement set-up. (b) SAR assessment methodology from the temperature rise. (c) Simulated and measured SAR results.

\section{Antennas for off-body communications at millimeter-waves}

At microwaves, it is widely accepted that antennas placed in close proximity to a lossy medium experience strong power absorption, radiation pattern distortion, shift in resonance frequency, and changes in the input impedance, e.g. [1],[19]-

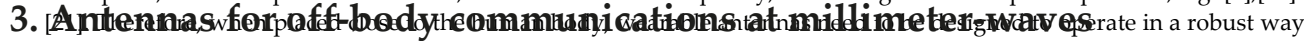
so that the influence of the body on the antenna performance is minimized. Patch antennas have been identified as one of the best solutions for off-body communications [1]. These are simple and low-cost structures, and their radiation at

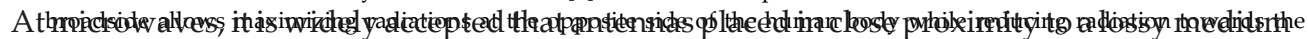
experyence strong power absorption, radiation pattern distortion, shift in resonance frequency,

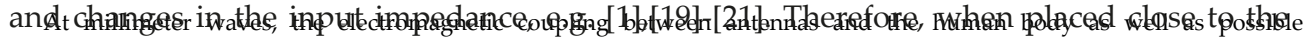

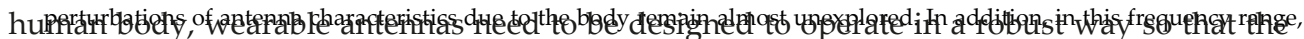
influence of the body on the antenna performance is minimized. Patch antennas have been

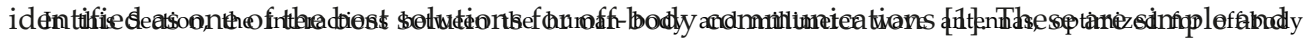

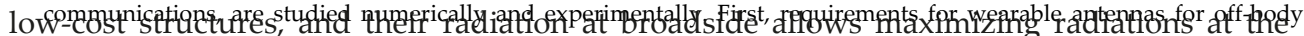
communications are briefly outlined. Then, the influence of the antenna feeding is investigated. Then, a four-patch

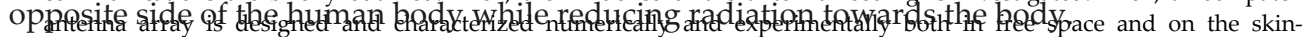
equivalent phantom described in the previous section. SAR and incident power density distributions on the phantom are

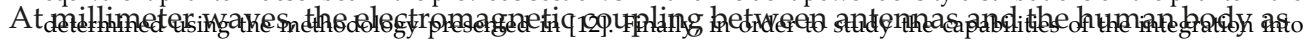

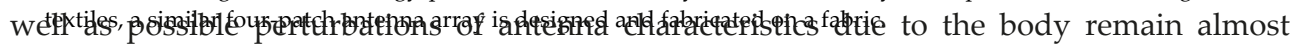


unexplored. In addition, in this frequency range, a particular attention must be paid to the power absorbed in the body since this absorption is very localized.

In this Section, the interactions between the human body and millimeter wave antennas, optimized for off-body communications, are studied numerically and experimentally. First, requirements for wearable antennas for off-body communications are briefly outlined. Then, the influence of the antenna feeding is investigated. Then, a four-patch antenna array is designed and characterized numerically and experimentally both in free space and on the skinequivalent phantom described in the previous section. SAR and incident power density distributions on the phantom are determined using the methodology presented in [12]. Finally, in order to study the capabilities of the integration into textiles, a similar four-patch antenna array is designed and fabricated on a fabric.

\subsection{Antenna requirements for off-body communications}

Wearable antennas have to be as compact as possible to be integrated with the transceiver. They have to be efficient with minimal power absorption inside the human body that behaves as a highly lossy dispersive dielectric material at millimeter waves. The antennas also have to be light weight and, in some particular cases, conformable to the human body shape. Because of the high atmospheric attenuation at $60 \mathrm{GHz}$ and limitations on the radiated power, mediumgain antennas $(\sim 12 \mathrm{dBi})$ are often required [14]. Indeed, in controlled environments, line-ofsight (LOS) channels can be efficiently exploited using medium-gain passive antennas, whereas directive beam steering antennas are desirable for non-line-of-sight (NLOS) channels so as to comply with the power link budgets [14]-[18]. In our studies, we only consider LOS scenarios and thus restrict our consideration to passive medium-gain antennas.

\subsection{Influence of the antenna feeding}

The influence of the antenna feeding is investigated when the antenna is placed on the human body. At lower frequencies, patch antennas have been presented as the best solution for offbody communications. However, at millimeter waves, the influence of spurious waves due to the feeding lines on radiating patterns cannot be neglected. That is why, multilayer antenna designs are generally considered in order to overcome this issue.

The interaction with the human body and two types of patch antennas is studied: (1) a linearlypolarized antenna and (2) a linearly-polarized aperture coupled patch antenna. These antennas are printed on a $0.127 \mathrm{~mm}$-thick RT Duroid 5880 substrate $\left(h=127 \mu \mathrm{m}, \varepsilon_{r}=2.2\right.$, $\left.\tan \delta=0.003\right)$.

\subsubsection{Microstrip patch antenna}

A simple patch antenna is optimized to achieve a maximum gain at $60 \mathrm{GHz}$. The dimensions are given in Fig. 7. The reflection coefficient and radiation patterns are studied numerically in free space and on the human body when the antenna is placed at $1 \mathrm{~mm}$ above the phantom. For the numerical modeling, a parallelepipedic $10 \times 100 \times 100 \mathrm{~mm}^{3}$ phantom is used and a Debye model has been used to express the complex permittivity $\varepsilon^{*}$ of the skin-equivalent phantom (see Section 2). 
The reflection coefficient is very slightly affected by the human body (Fig. 8) and the radiation pattern remains stable at the opposite side of the human body, whereas the backward radiations are highly reduced in H-plane (Fig. 9). These results demonstrate that microstrip patch antennas are only slightly sensitive to the human body proximity at $60 \mathrm{GHz}$.

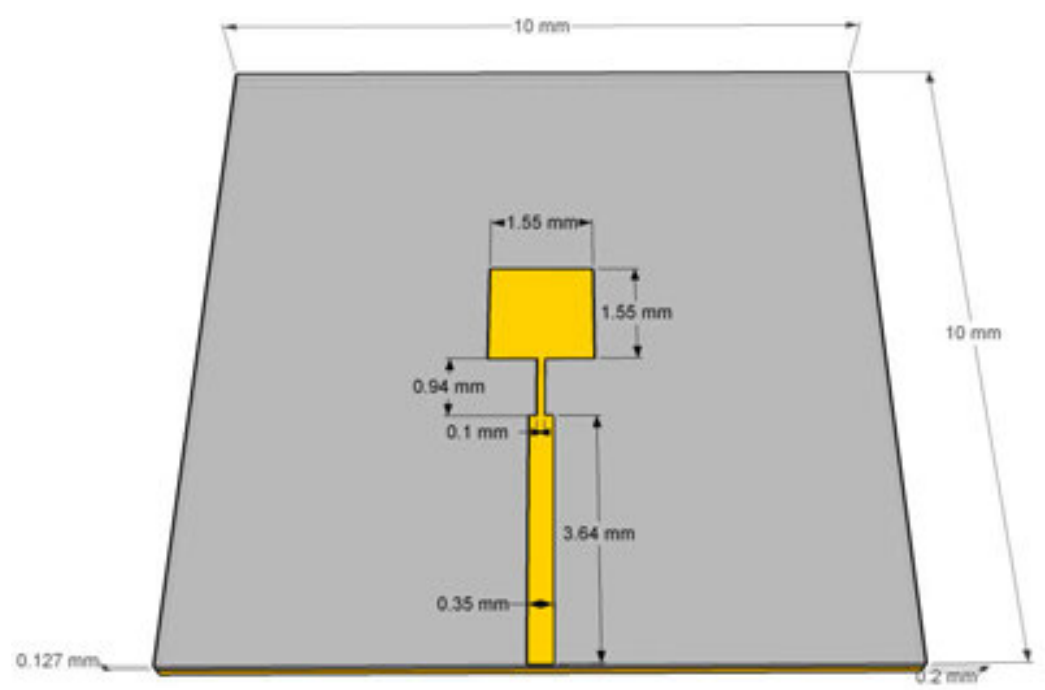

Figure 7. Microstrip patch antenna at $60 \mathrm{GHz}$.

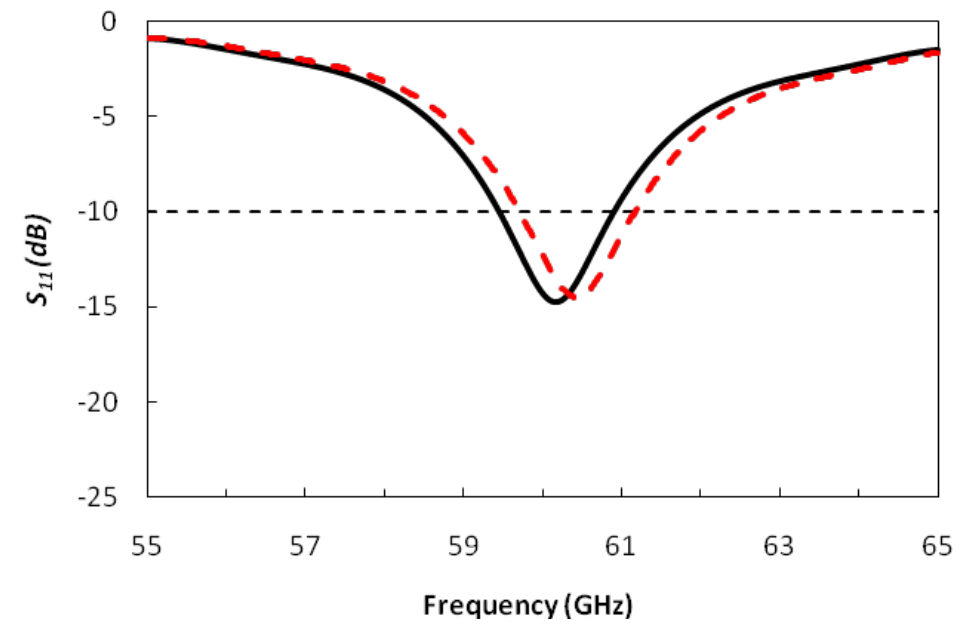

Figure 8. Simulated reflection coefficient of the microstrip patch antenna. —— In free space. _ — On the skin-equivalent phantom. 


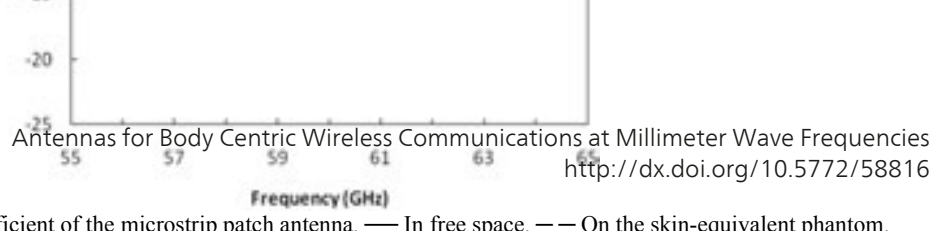

Fig.8. Simulated reflection coefficient of the microstrip patch antenna. - In free space. - - On the skin-equivalent phantom.

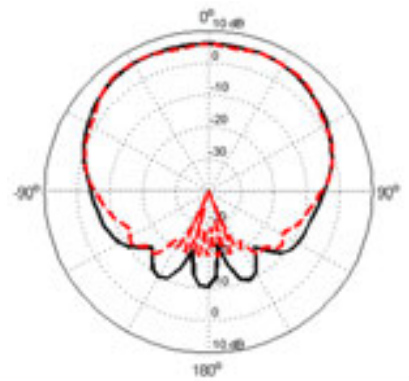

(a) $E$-plane

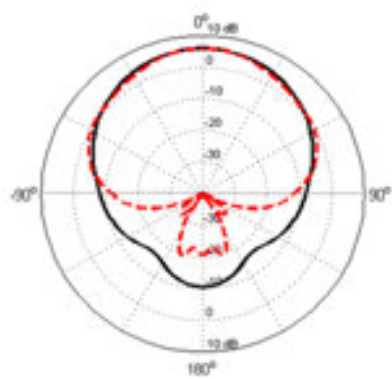

(b) $H$-plane

Fig.9. Simulated radiation pattern of the microstrip patch antenna. — In free space. - - On the skin-equivalent phantom.

Figure 9. Simulated radiation pattern of the microstrip patch antenna. — In free space. — — On the skin-equivalent pB.2.20m.Aperture coupled patch antenna

Fig. 10 shows the configuration of the aperture-coupled patch antenna (ACPA). The slot is optimized to $0.26 \times 1 \mathrm{~mm}^{2}$ for maximum coupling with a stub length of $0.34 \mathrm{~mm}$. In order to consider the easiness of implementation, a 0.2 -mm-thick ground plane is

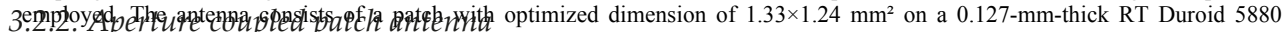
substrate. Low thickness and low-permittivity substrate are used for reducing surface waves.

The reflection coefficient $S_{11}$ (Fig.11) and radiation patterns (Fig.12) are investigated in free space and on the skin-equivalent Fijgant $\$$ Q the ACPA. However as far as the radiation pattern is concerned, the backward radiations are highly reducesd (i.e. by at least $10 \mathrm{~dB}$ ).

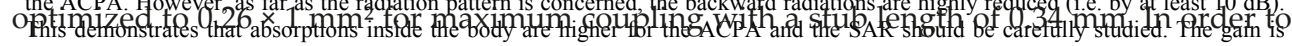

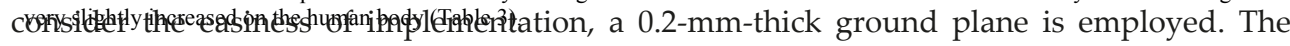
antenna consists of a patch with optimized dimension of $1.33 \times 1.24 \mathrm{~mm}^{2}$ on a 0.127 -mm-thick RT Duroid 5880 substrate. Low thickness and low-permittivity substrate are used for reducing surface waves.

The reflection coefficient $S_{11}$ (Fig. 11) and radiation patterns (Fig. 12) are investigated in free space and on the skin-equivalent phantom (antenna/body spacing $\mathrm{h}=1 \mathrm{~mm}$ ). It is worthwhile to note that the $S_{11}$ is even less affected by the human body proximity for the ACPA. However, as far as the radiation pattern is concerned, the backward radiations are highly reduced (i.e. by at least $10 \mathrm{~dB}$ ). This dempanstrates that absorptions inside the body are(bigher for the ACPA

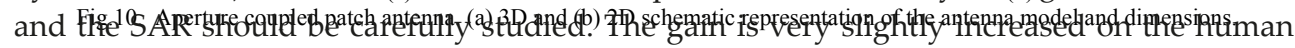
body (Table 3).

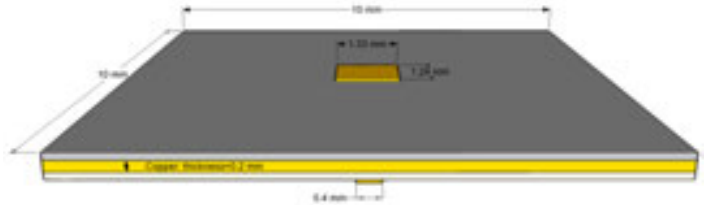

(a)

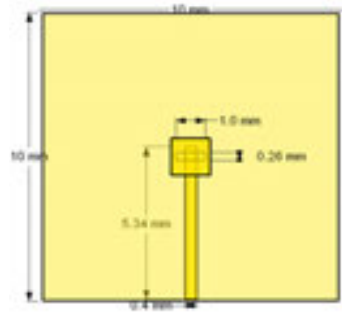

(b)

Figure 10. Aperture coupled patch antenna. (a) 3D and (b) 2D schematic representation of the antenna model and dimensions.

Figure 10. Aperture coupled patch antenna. (a) 3D and (b) 2D schematic representation of the antenna model and dimensions.

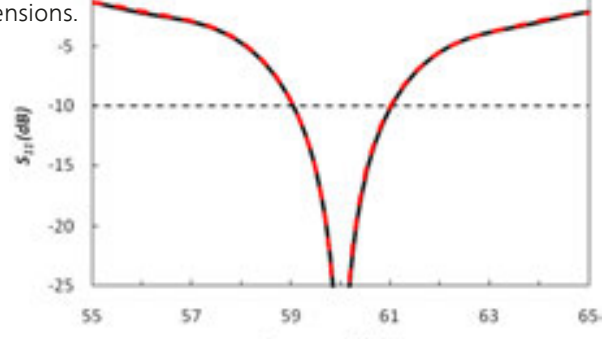


Figure 11. Simulated reflection skin-equivalent phantom.

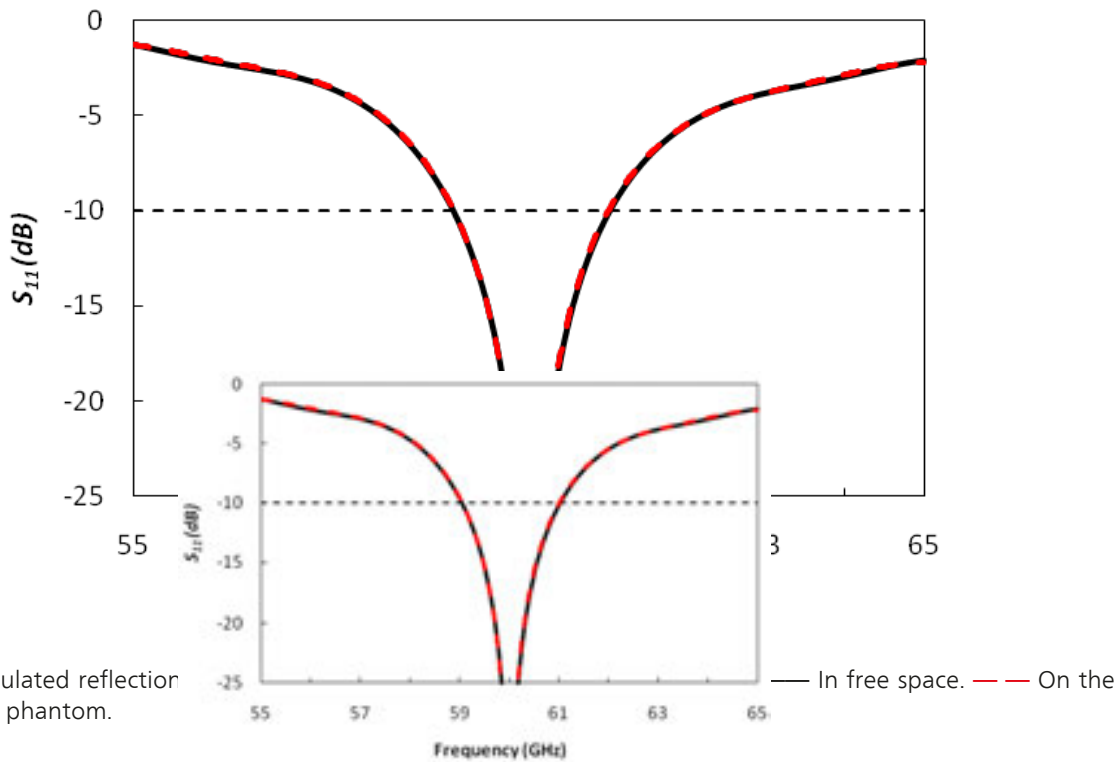

Fig.11. Simulated reflection coefficient of the aperture coupled patch antenna. — In free space. - On the skinequivalent phantom.

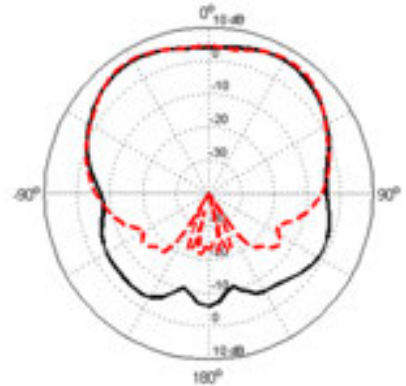

(a) E-plane

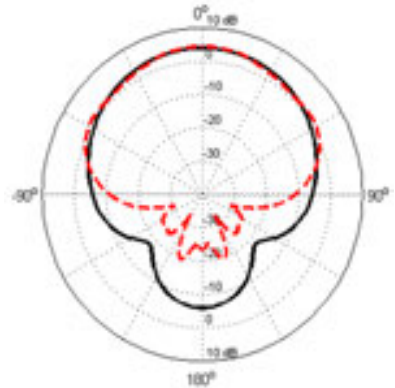

(b) $H$-plane

Fig.12. Simulated radiation pattern of the aperture coupled patch antenna. - In free space. -- On the skin-equivalent phantom.

$\mathbf{F}^{3} \mathbf{2}^{3}{ }_{12}$ Specific Absorption Rate, (SAR) comparison

In free space _ _ on the skinThe SAR are compared for the microstrip patch antenna and ACPA for an antenna/body spacing $h=1 \mathrm{~mm}$ and for an incident power of 1 W. The peak SAR obtained for the ACPA is 41 times higher compared to that obtained with the microstrip patch antenna. Therefore, it is not recommended to use ACPA because the input power would be highly limited compared to that of the microstrip patch antenna to comply with the exposure limits [22], resulting in a lower link budget (gain and efficiency remain almost equivalent 3for.bothsprexifias) Absorption Rate (SAR) comparison

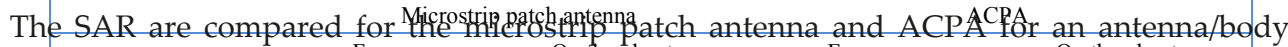

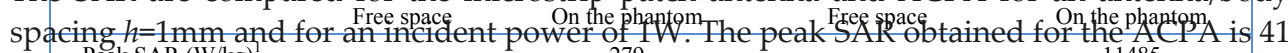
Peak SAR (W/kg)
times higher compared to that obtained with the microstrip patch antenna. Therefore, it is not Peak gain (dBi)
recommend

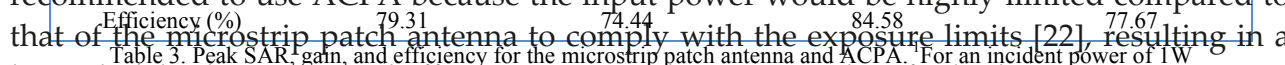
lower link budget (gain and efficiency remain almost equivalent for both antennas).

\subsubsection{Conclusion}

Two patch antennas have been compared numerically in free space and on a skin-equivalent phantom. For the microstrip antenna and ACPA, the influence of the human body is very weak, and their performances remain stable. However, the SAR resulting from the ACPA is 41 times higher compared to that obtained with the microstrip antenna. Therefore, it is highly recommended to avoid aperture coupled feeds. If it is necessary, the feeding line could be sandwiched between two substrates with top and bottom grounds [23]. 


\begin{tabular}{ccccc}
\hline & \multicolumn{2}{c}{ Microstrip patch antenna } & \multicolumn{2}{c}{ ACPA } \\
\hline & Free space & On the phantom & Free space & On the phantom \\
\hline Peak SAR $(\mathrm{W} / \mathrm{kg})^{1}$ & - & 279 & - & 11485 \\
\hline Peak gain $(\mathrm{dBi})$ & 6.01 & 6.03 & 6.22 & 6.70 \\
\hline Efficiency $(\%)$ & 79.31 & 74.44 & 84.58 & 77.67 \\
\hline
\end{tabular}

Table 3. Peak SAR, gain, and efficiency for the microstrip patch antenna and ACPA. ${ }^{1}$ For an incident power of $1 \mathrm{~W}$

\subsubsection{Conclusion}

Two patch antennas have been compared numerically in free space and on a skin-equivalent phantom. For the microstrip antenna and ACPA, the influence of the human body is very weak, and their performances remain stable. However, the SAR resulting from the ACPA is 41 times higher compared to that obtained with the microstrip antenna. Therefore, it is highly recommended to avoid aperture coupled feeds. If it is necessary, the feeding line could be sandwiched between two substrates with top and bottom grounds [23].

\subsection{Patch antenna array}

\subsubsection{Antenna model}

To satisfy the criteria summarized in Section 3.1 and following the conclusions drawn in Section 3.2, a microstrip-fed four-patch single-layer antenna array has been chosen [24]. It is printed on a thin RT Duroid 5880 substrate $\left(h=127 \mu \mathrm{m}, \varepsilon_{\mathrm{r}}=2.2\right.$, $\left.\tan \delta=0.003\right)$. The layout is represented in Fig. 13a. A single rectangular patch antenna typically provides a $7 \mathrm{dBi}$ gain; a $2 \times 2$ antenna array is chosen here to reach a gain of $12 \mathrm{dBi}$ with about the same beamwidth in E-and H-planes. The inter-element spacing is selected to achieve a good trade-off between high gain and low side lobes. Similar $2 \times 2$ antenna arrays have already been reported in a multilayer configuration [25] or fed by a coaxial probe [26],[27], which would make them difficult to fabricate on flexible or textile substrates. Hence, here all patches are fed using a single-layer corporate feed network. The antenna is linearly-polarized along y-direction, and, for meas-

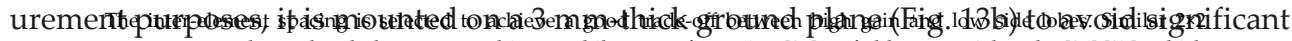

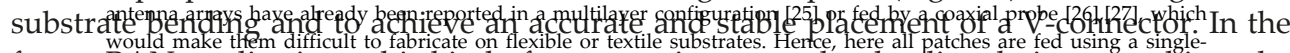

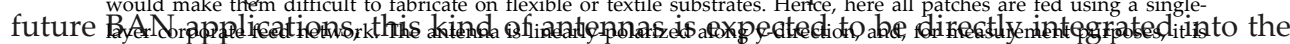

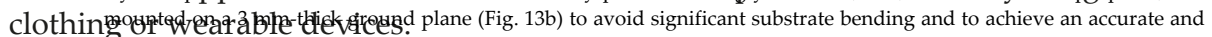
stable placement of a V-connector. In the future BAN applications, this kind of antennas is expected to be directly integrated into the clothing or wearable devices.

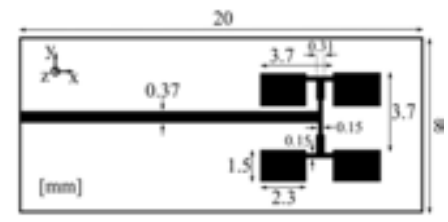

(a)

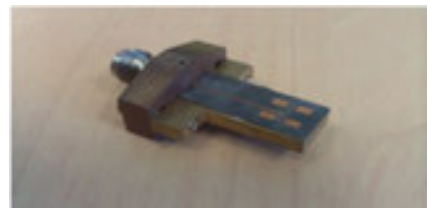

(b)

Figure 13. 2×2-patch single-layer antenna array at $60 \mathrm{GHz}$ [24]. (a) Schematic representation of the antenna model and dimensions. (b) Manufactured antenna with a V-connector.

Figure 13. Patch single-layer antenna array at $60 \mathrm{GHz}$ [24]. (a) Schematic representation of the antenna model and

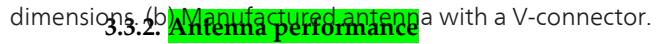

The antenna reflection coefficient $S_{11}$ was measured in free space and on the skin-equivalent phantom (Fig. 14). It remains below $-10 \mathrm{~dB}$ from $59 \mathrm{GHz}$ to $65 \mathrm{GHz}$. It is clear that the skin-equivalent phantom does not affect the antenna reflection coefficient. This is not the case at microwaves where a resonance shift is usually observed.

In addition, the radiation patterns in E- and H-planes are plotted in Fig. 15 at $60 \mathrm{GHz}$. The gain was measured by the comparison method with a 20-dBi standard horn, and the directivity is found from a 3D radiation pattern measurement. It can be seen in Fig. 15 that front radiations remain very slightly affected.

The backward radiation was measured separately in both configurations. Whereas the measured level on the phantom is mainly reduced in the E-plane (Fig. 15c) due to the absorption and reflection, it remains very slightly affected in the $H$ - 


\subsubsection{Antenna performance}

The antenna reflection coefficient $S_{11}$ was measured in free space and on the skin-equivalent phantom (Fig. 14). It remains below-10 dB from $59 \mathrm{GHz}$ to $65 \mathrm{GHz}$. It is clear that the skinequivalent phantom does not affect the antenna reflection coefficient. This is not the case at microwaves where a resonance shift is usually observed.

In addition, the radiation patterns in E-and H-planes are plotted in Fig. 15 at $60 \mathrm{GHz}$. The gain was measured by the comparison method with a $20-\mathrm{dBi}$ standard horn, and the directivity is found from a 3D radiation pattern measurement. It can be seen in Fig. 15 that front radiations remain very slightly affected.

The backward radiation was measured separately in both configurations. Whereas the measured level on the phantom is mainly reduced in the E-plane (Fig. 15c) due to the absorption and reflection, it remains very slightly affected in the $H$-plane (Fig. 15d). This could be expected since the absorption is higher when the $E$-field is parallel to the phantom surface [28]. These observations are in agreement with the calculated and measured SAR and incident power density (IPD) as shown in Fig. 16. More details regarding the measurement methodology can be found in [12].

At this frequency, the measured gains in free space and on the phantom equal $11.8 \mathrm{dBi}( \pm 0.3 \mathrm{~dB})$ and $11.9 \mathrm{dBi}( \pm 0.3 \mathrm{~dB})$, respectively. This demonstrates the small effect of the phantom presence. At $60 \mathrm{GHz}$, the measured directivity was assessed to be equal to $13.9 \mathrm{dBi}( \pm 0.3 \mathrm{~dB})$ and $14.1 \mathrm{dBi}$ $( \pm 0.3 \mathrm{~dB})$, respectively. Comparison of the measured directivities with the measured gains leads to antenna efficiencies of $62 \%$ and $60 \%$, respectively. This efficiency value is typical in V-band for this kind of antennas and could be further improved, for instance using a fused quartz substrate [25] instead of RT Duroid 5880. Whereas the antenna efficiency at microwaves can be strongly affected by the body presence even for patch antennas [1], it is found here that it remains stable in V-band.

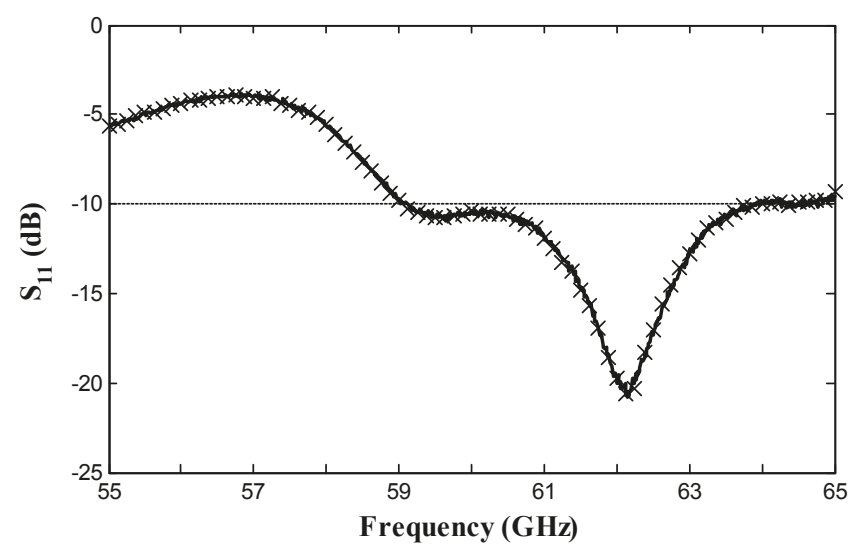

Figure 14. Measured reflection coefficient of the antenna. - - - In free space. $x \times x$ On the skin-equivalent phantom. 


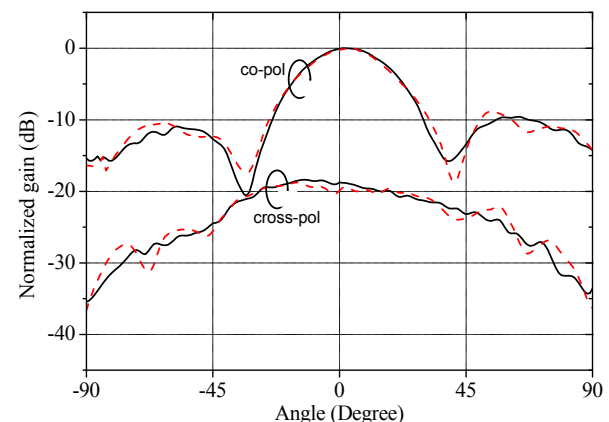

(a) E-plane (front radiation)

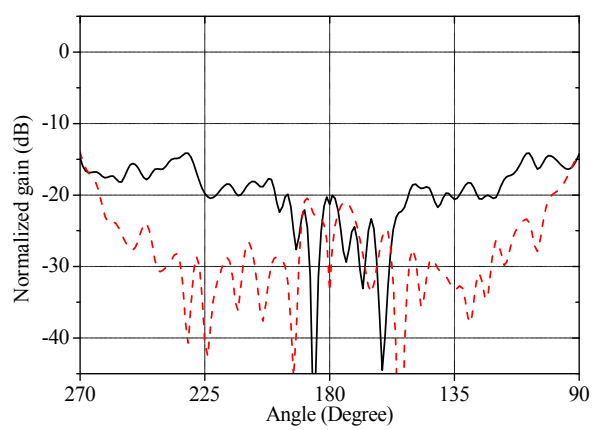

(c) E-plane (back radiation: co-pol only)

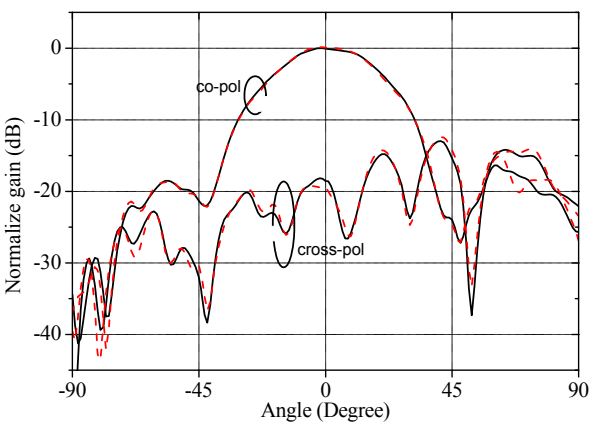

(b) $H$-plane (front radiation)

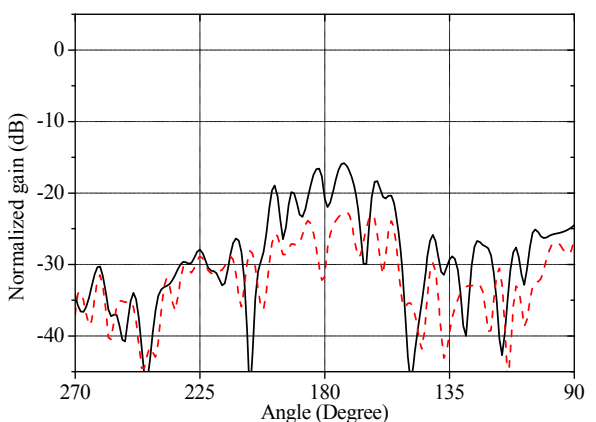

(d) $H$-plane (back radiation: co-pol only)

Fig.15. Measured normalized radiation patterns at $60 \mathrm{GHz}$ in $E$ - and $H$-planes. — Measurement in free space. - - - Measurement on the phantom.

Figure 15. Measured normalized radiation patterns at60 GHzino $E$-and $\mathrm{H}$-planes. —— Measurement in free space. ---

Measurement on the phantom
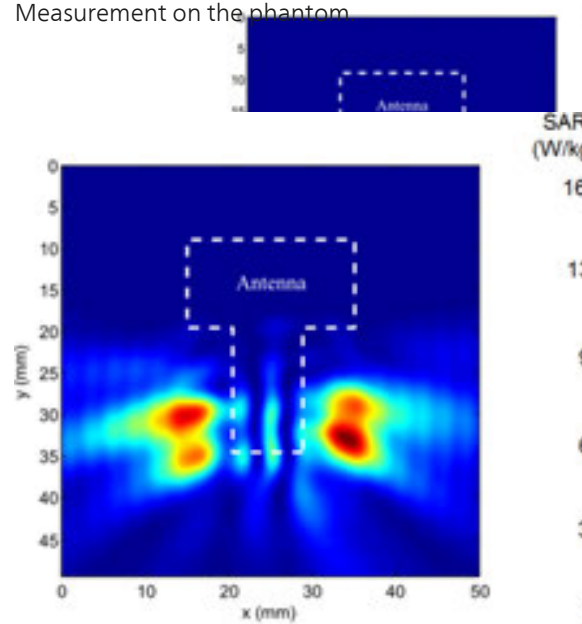
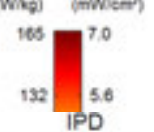

(W/kg)

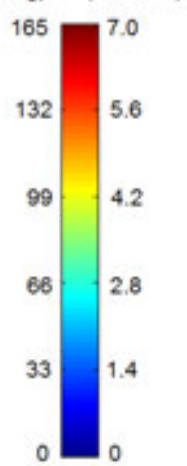

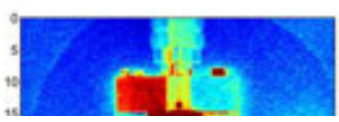

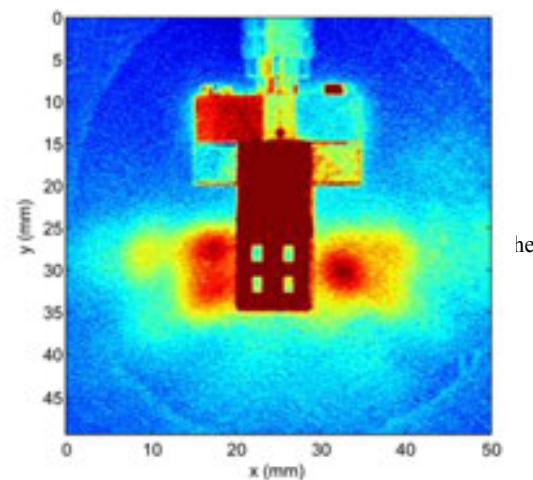

Figure 16. SAR and IPD distributions at $60 \mathrm{GHz}$. (Left) Numerical results for the antenna on the skin. (Right) Measurements on the skin-equivalent phantom. $P_{\text {in }}=322 \mathrm{~mW}$. 


\subsection{Textile antennas}

Textile antennas at millimeter waves could be of great interest for many applications. However, on-textile fabrication process is very challenging at these frequencies, especially due to the roughness of the textile surface and the size of textile fibers and electrotextiles with respect to the geometrical dimensions of the metallic patterns.

It was demonstrated in [29] that commercial textiles can be used as antenna substrates at millimeter waves. Some results are presented here showing a $60-\mathrm{GHz}$ textile-based antenna for off-body wireless communications with the ability to be bent and deformed into an arbitrary shape. A simple, but representative patch antenna array is fabricated using an adhoc manufacturing process. Compared to the antenna presented in Section 3.3, this results in a highly flexible antenna.

\subsubsection{Technological fabrication process}

The fabrication process of millimeter-wave textile antennas has been presented in [29] and [31] (Fig. 17). The first step (Fig. 17a) consists in placing an electrotextile layer (e.g. ShieldIt Super) on the lower side of the textile (ground plane), and flexible copper foil on the top side. The second step (Fig. 17b) consists in micromachining the copper foil using a laser machine with optimized laser parameters to avoid any damage of the textile substrate such as ragged or burnt edges.

Hence, using a laser machine (ProtoLaser S, LPKF, OR, USA) operating at 1064nm with a pulse duration of $7.5 \mathrm{~ns}$ and a spot size equals $25 \mu \mathrm{m}$, the laser parameters were optimized. A laser fluence of $24.4 \mathrm{~mJ} / \mathrm{cm}^{2}$ with three cycles on the surface of the substrate has been used for the copper foil ablation (repetition rate $=75 \mathrm{kHz}$, power= $16.0 \mathrm{~W}$ ) without affecting the textile substrate. These fabrication conditions lead to a geometrical accuracy of about $10 \mu \mathrm{m}$. It is worthwhile to underline that the accuracy reported so far with two conductive fabrics, namely knitted P130 and woven Nora fabric, is only about $\pm 0.5 \mathrm{~mm}$ and $\pm 0.15 \mathrm{~mm}$, respectively [32]. Finally, the last step (Fig. 17c) consists in manually removing the unwanted parts of the copper foil from the surface of the textile.

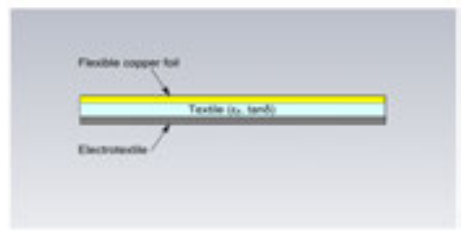

(a) Substrate preparation

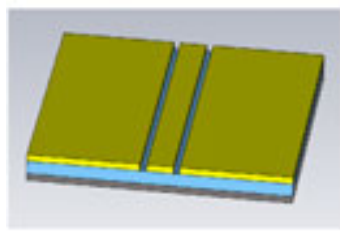

(b) Laser ablation

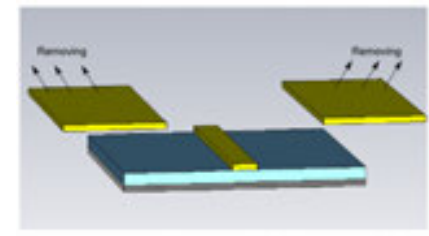

(c) Removing of undesired parts

Figure 17. Main technological steps for the manufacturing of printed circuits and antennas on textiles in V-band.

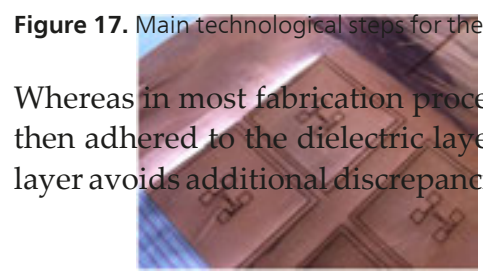

(a)

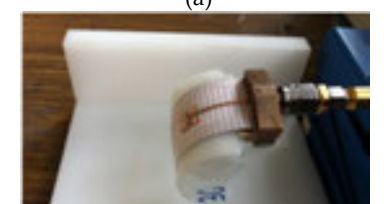

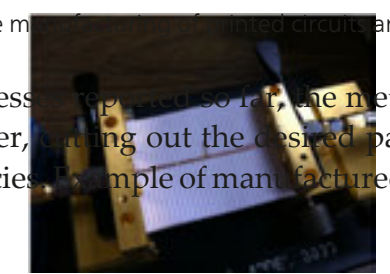

(b)

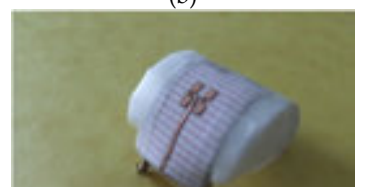

pnd antennas on textiles in V-band.

etallic part is cut separately and pattern directly on the dielectric ed microstrip antennas and lines

(c) 


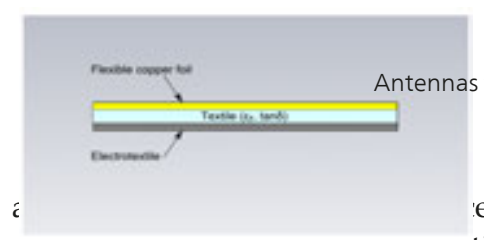

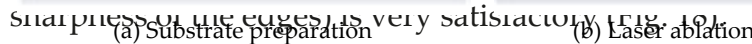

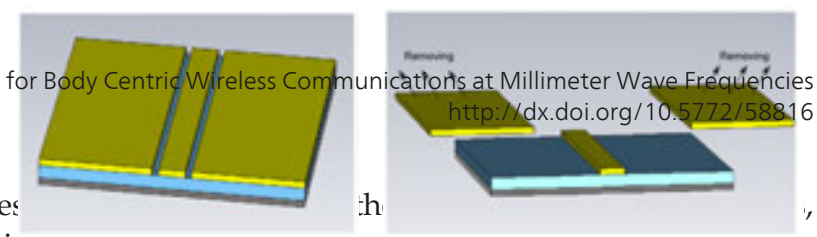

(c) Removing of undesired parts

Figure 17. Main technological steps for the manufacturing of printed circuits and antennas on textiles in V-band.

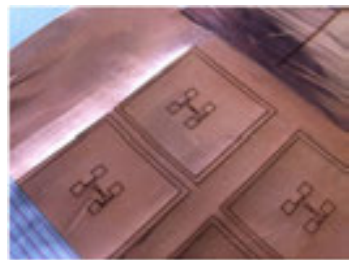

(a)

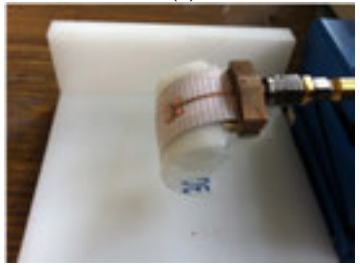

(d)

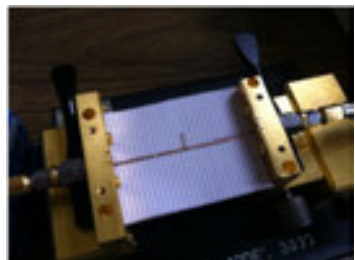

(b)

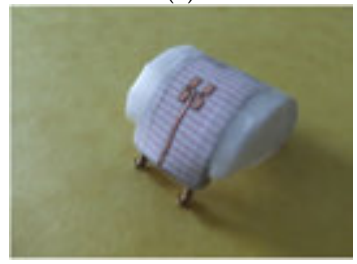

(e)

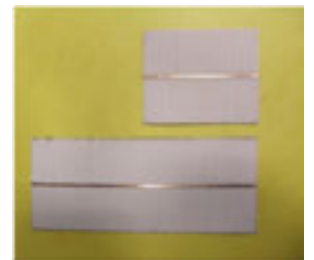

(c)

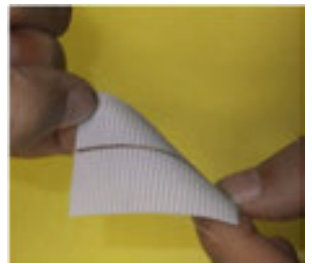

(f)

Figure 18. Examples of fabricated textile antennas and microstrip lines.

Figure 18. Examples of fabricated textile antennas and microstrip lines.

\subsubsection{Textile characterization}

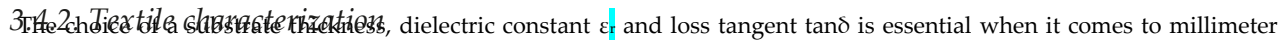
waves in order to avoid losses and also to enhance the efficiency. The methodology employed to retrieve the dielectric

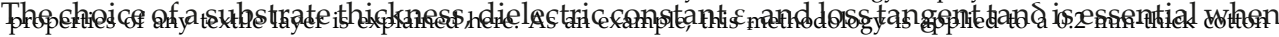

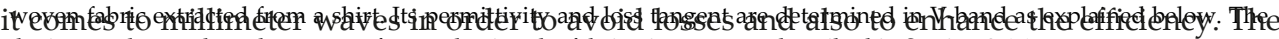
devifes ynder test have peen manufactured using the fabrication process described in Section $3^{3} f^{1}{ }^{1}$ layer is explained

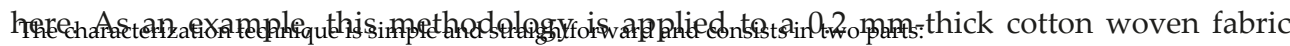
extracted from a shirt. Its permittivity and loss tangent are determined in V-band as explained

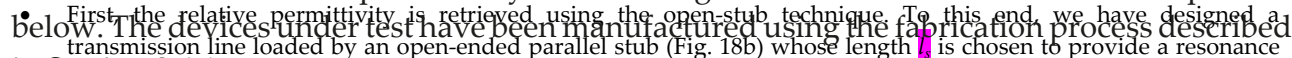
in Seftiotz $304 \mathrm{GH}$. The resonant frequency is measured in transmission with a V-band Anritsu universal test fixture $3680 \mathrm{~V}$ (Fig. 18b) connected to.an Agịlent 8510XF vector network analyzer (VNA). The measurement set-up has been

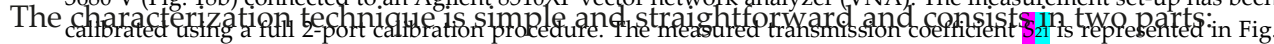

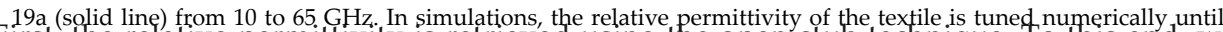

- First the relative permittivitith is retrieved using the open-stub technique. To this end, we have designed a transmission line loaded by an open-ended parallel stub (Fig. 18b) whose

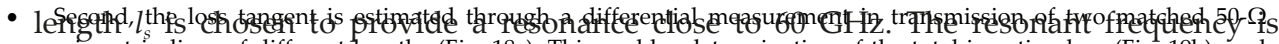

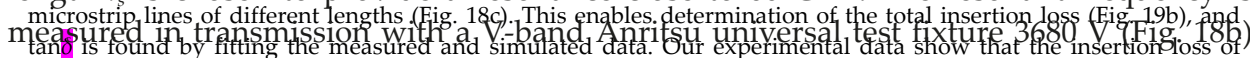

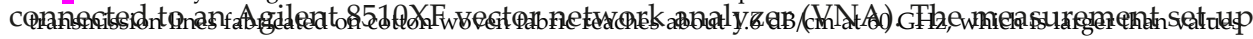

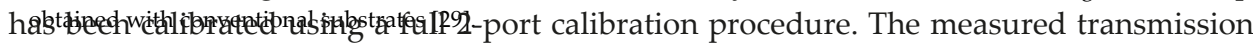
coefficient $S_{21}$ is represented in Fig. 19a (solid line) from 10 to $65 \mathrm{GHz}$. In simulations, the relative permittivity of the textile is tuned numerically until the theoretical $S_{21}$ curve coincides with the measured one.

- Second, the loss tangent is estimated through a differential measurement in transmission of two matched 50- $\Omega$ microstrip lines of different lengths (Fig. 18c). This enables determination of the total insertion loss (Fig. 19b), and $\tan \delta$ is found by fitting the measured and simulated 
data. Our experimental data show that the insertion loss of transmission lines fabricated on cotton woven fabric reaches about $1.6 \mathrm{~dB} / \mathrm{cm}$ at $60 \mathrm{GHz}$, which is larger than values obtained with conventional substrates [29].

The best agreement between simulations and experiments is obtained with $\varepsilon_{\mathrm{r}}=2.0$ and $\tan \delta=0.02$. These values will be used for the antenna design. Since commercial textiles are lossy, a slight deviation in the determination of their loss-tangent would have a minor impact. Therefore, deviations due to the use of electromagnetic software are considered as acceptable.

The insertion loss of a $50-\Omega$ microstrip line printed on a $0.2 \mathrm{~mm}$-thick textile is about 1.6 $\mathrm{dB} / \mathrm{cm}$, which is quite important compared to conventional substrates such as RT Duroid 5880, fused quartz and alumina [29]. However, these substrates are not as flexible as textiles. For a fair comparison, we should consider a flexible substrate such as PDMS where the insertion losses are much more important ( 3 dB/cm for a 0.2mm-thick PDMS) [30].

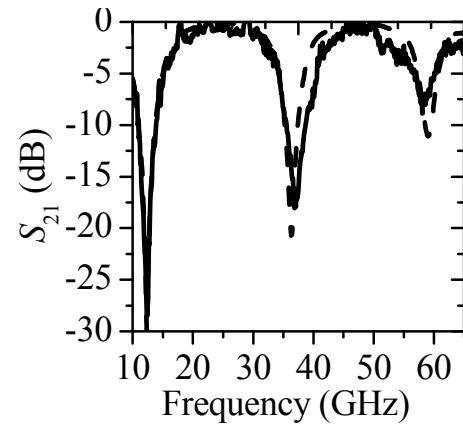

(a)

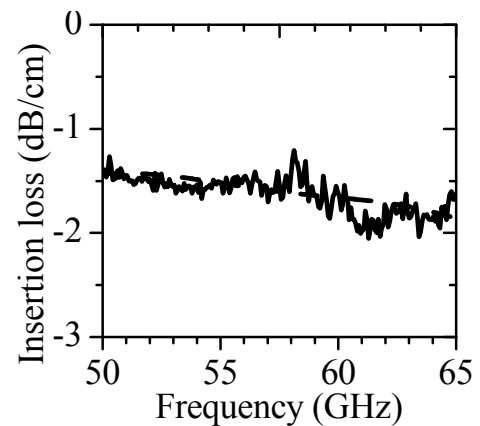

(b)

Figure 19. (a) Transmission coefficient $S_{21}$ of the stub loaded microstrip line $(\underline{l}=4.58 \mathrm{~mm}, \boldsymbol{L}=50 \mathrm{~mm}$. (b) Insertion loss of a $50 \Omega$ line. The

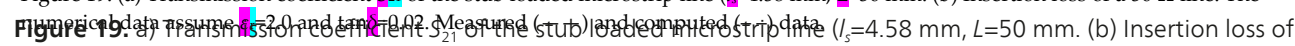
a $50 \Omega$ line. The numerical data assume $\varepsilon_{\mathrm{r}}=2.0$ and $\tan \delta=0.02$. Measured $(--)$ and computed $(--)$ data.

\subsubsection{Microstrip patch antenna}

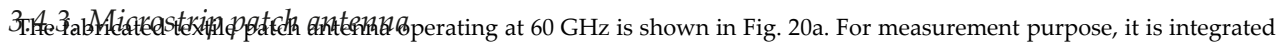
with a V-connector. The flexibility of the antenna is demonstrated in Fig. 20b. The antenna was optimized to operate at

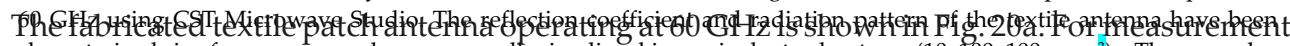

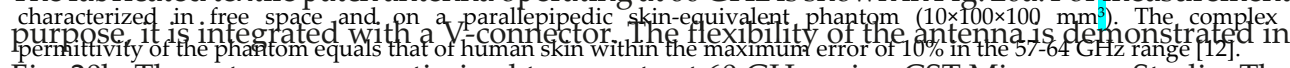
Fig. 20b. The antenna was optimized to operate at $60 \mathrm{GHz}$ using CST Microwave Studio. The

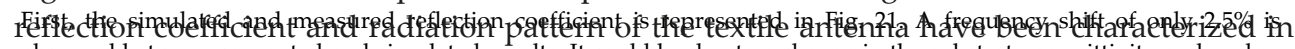
observed between computed and simulated results. It could pe due to a change in the substrate permittivity and under-

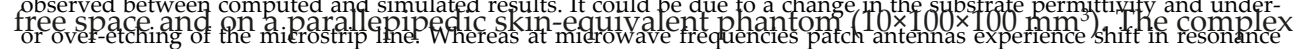

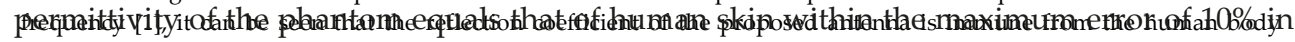
tprexisyitr $6 \mathrm{GHz}$ range [12].

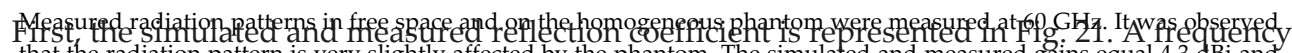
that the radiation pattern is very slightly affected by the phantom. The simulated and measured gains equal $4.3 \mathrm{dBi}$ and

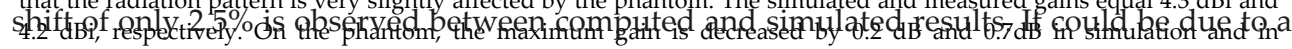

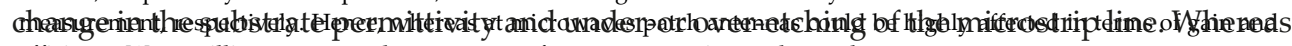

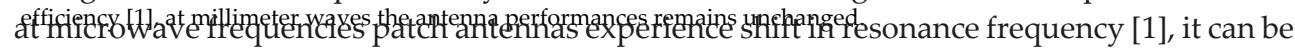
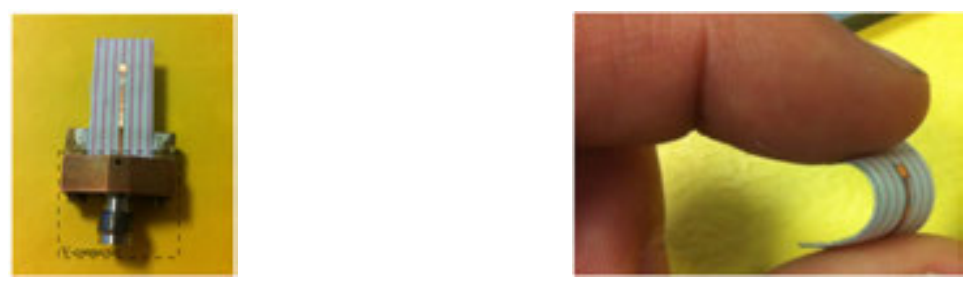


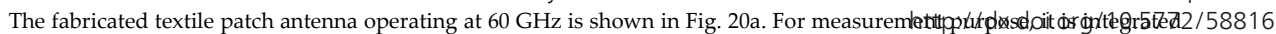
with a V-connector. The flexibility of the antenna is demonstrated in Fig. 20b. The antenna was optimized to operate at $60 \mathrm{GHz}$ using CST Microwave Studio. The reflection coefficient and radiation pattern of the textile antenna have been

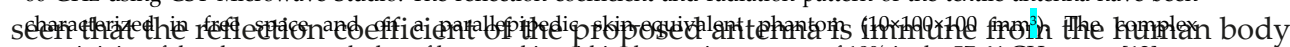
permittivity of the phantom equals that of human skin within the maximum error of $10 \%$ in the $57-64 \mathrm{GHz}$ range [12]. proximity.

First, the simulated and measured reflection coefficient is represented in Fig. 21. A frequency shift of only $2.5 \%$ is

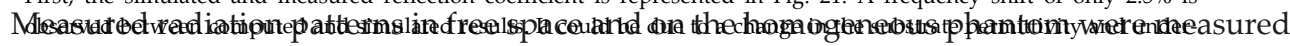

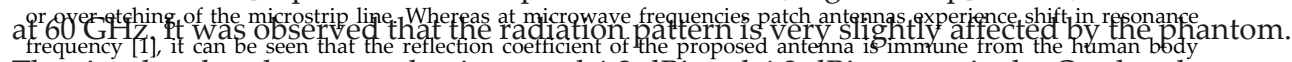
Tperingulated and measured gains equal $4.3 \mathrm{dBi}$ and $4.2 \mathrm{dBi}$, respectively. On the phantom, the maximum gain is decreased by $0.2 \mathrm{~dB}$ and $0.7 \mathrm{~dB}$ in simulation and in measurement,

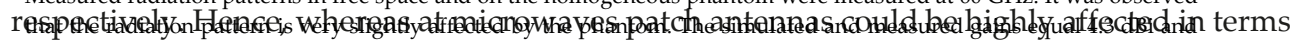

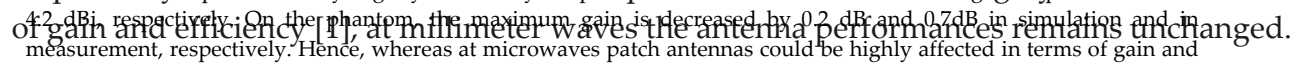
efficiency [1], at millimeter waves the antenna performances remains unchanged.

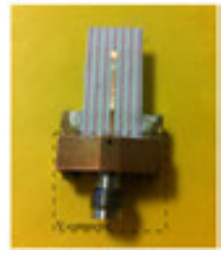

(a)

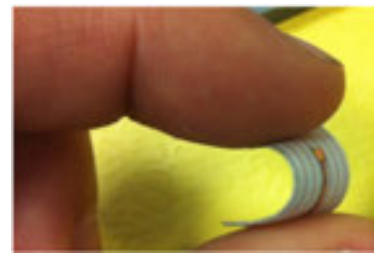

(b)

Figure 20. Photography of the fabricated patch antenna with a V-connector.

Figure 20. Photography of the fabrieated patch antenna with a V-connector.

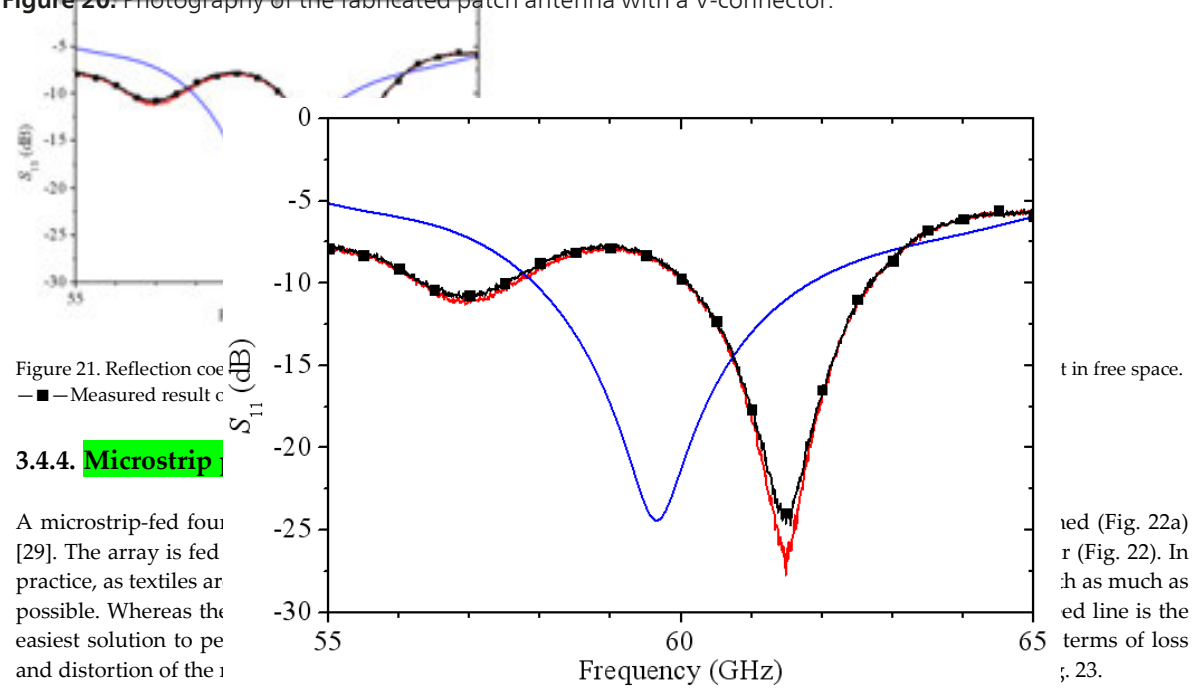

Figure 21. Reflection coefficient of the wearable patch antenna. ——Computed result in free space.— Measured result in free space.

-Measured result on the skin-equivalent phantom.

\subsubsection{Microstrip patch antenna array}

A microstrip-fed four-patch single-layer antenna array printed on the $0.2 \mathrm{~mm}$-thick textile has been designed (Fig. 22a) [29]. The array is fed by a 15.2mm-long microstrip line to avoid too 
strong reflections from the V-connector (Fig. 22). In practice, as textiles are more lossy than classical substrates, it is recommended to reduce the access line length as much as possible. Whereas the antenna could be fed using a central probe, (as shown in Fig. 22b), the microstrip feed line is the easiest solution to perform measurements on textile. We will discuss the impact of this microstrip line in terms of loss and distortion of the radiation pattern. The fabricated antenna integrated with a V-connector is shown in Fig. 23.

Its reflection coefficient $S_{11}$ is measured using a 110-GHz Agilent 8510XF VNA and is shown in Fig. 24. Excellent agreement is obtained between simulated and measured results. The reproducibility of these results has been demonstrated and more information can be found in [29].

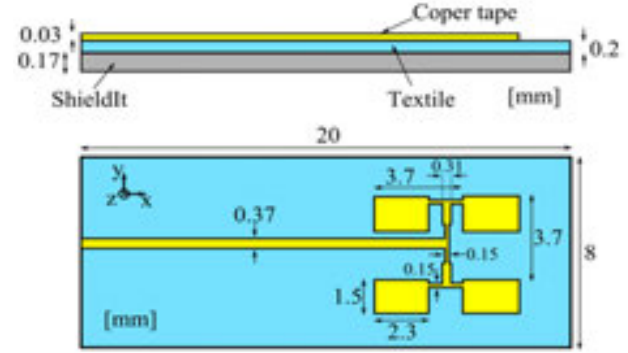

(a)

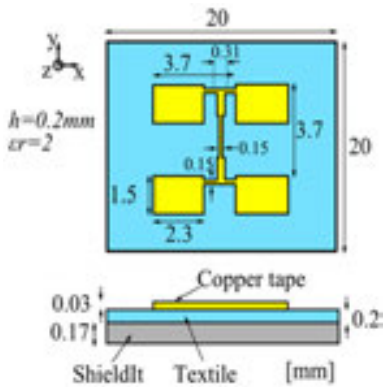

(b)

Figure 22. Layout of the microstrip antenna array printed on textile. (a) Antenna fed using a long microstrip line. (b) Antenna fed using

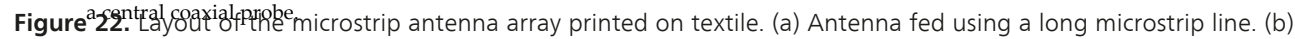
Antennafed using a central coaxiat

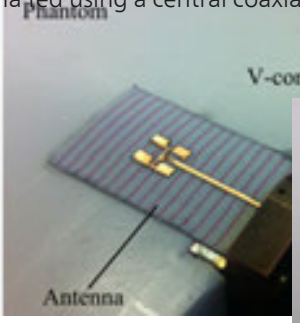

Figure 23. Measurement set-up $\mathrm{mm}$.

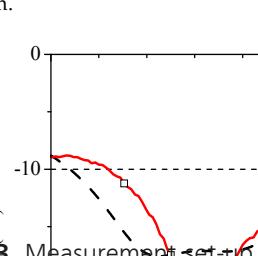

Figure $\frac{7}{23}$. Measurement set-t phantom eaqual to $d=0 \mathrm{~mm}$

The radiation patterns in E-and $\mathrm{H}$-plahes

The radiation patterns in E-and H-planes were measured in IETR's millimeter-wave anechoic chamber $30{ }_{55}$ the gain was measured by the eomparison method with a 20-dBi standard horn, and the directivity is foundeafrensf(f) 3D radiation pattern measurement. The co-polarization

Figure 24. Reflection coefficient of the microstrip antenna array printed on textile. $-\square-$ Measured. - - - Simulated.

The radiation patterns in E- and H-planes were measured in IETR's millimeter-wave anechoic chamber. The gain was measured by the comparison method with a 20-dBi standard horn, and the directivity is found from a 3D radiation pattern measurement. The co-polarization components measured in E- and H-planes at $60 \mathrm{GHz}$ are in a good agreement with the computed ones (Fig. 25). In E-plane, the non-symmetry of the co-polarization component is attributed to the spurious radiation of feeding lines whose width is larger compared to standard substrates at millimeter waves like RT Duroid 5880 (see Section 3.3), or other commonly used substrate such as fused quartz or Alumina. The main characteristics of these three different substrates are compared in [29] with those of the textile used here. These data 


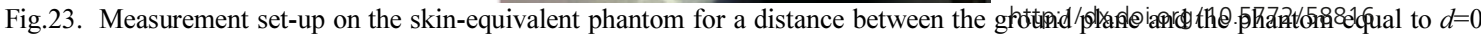
$\mathrm{mm}$.

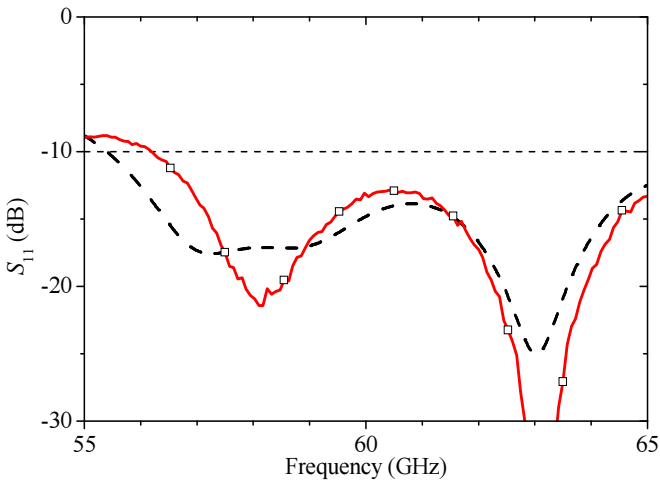

Fig.24. Reflection coefficient of the microstrip antenna array printed on textile. — $\square$ - Measured. - - Simulated.

Figure 24. Reflection coefficient of the microstrip antenna array printed on textile. $-\square-$ Measured.---Simulated.

The radiation patterns in E- and H-planes were measured in IETR's millimeter-wave anechoic chamber. The gain was measured by the comparison method with a $20-\mathrm{dBi}$ standard horn, and the directivity is found from a 3D radiation pattern measurement. The co-

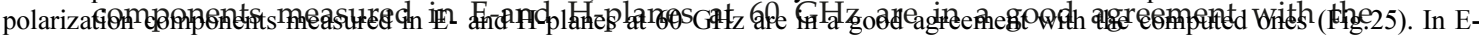

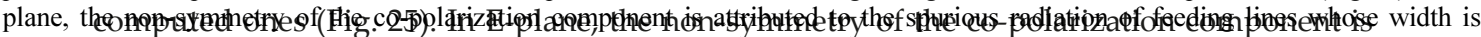
larger compared to standard substrates at millimeter waves like RT Duroid 588 (see Section 33) 3 or other commonly used substrate such as fused quartz or Alumina. The main characteristics of these three different substrates are compared in $\lceil 299$ with those of the

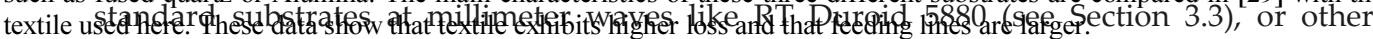

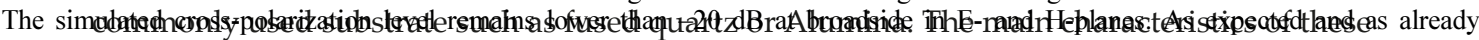

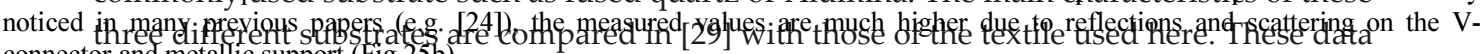
connector and metallic support (Fig.25b).

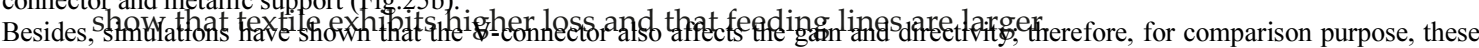
results are given for both configurations. (i.e. with and without connector). The cross-polarization level could be further improved using a multilayer antenna desigh, e.g. 251. However, as explained in Section 3.2, the at proter is not recommended for on-body

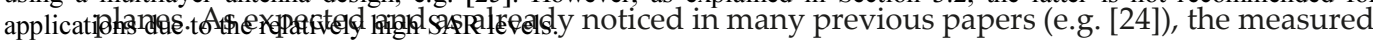

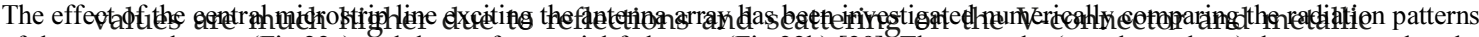
of the proposed array (Figr22a) and those of a coaxial-fed array (Fig.22b) [29]. These results (not shown here) demonstrate that the increase of the cross-pølarization levels and side lobe levels in E-plane is due to the main feed line.

In additiBesthegain, in the feed line (about $3.3 \mathrm{~dB}$ ). In order to increase the antenna gain and efficiency, the feed. line could be. shortened or even suppressedereforcib). for comparison purpose, these results are given for both configurations (i.e. With

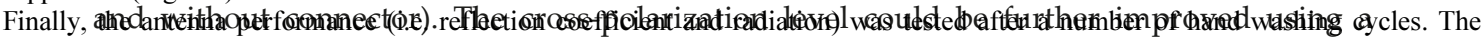

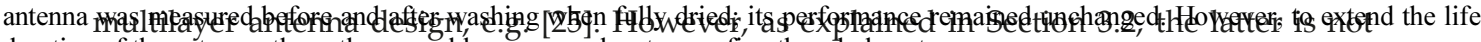

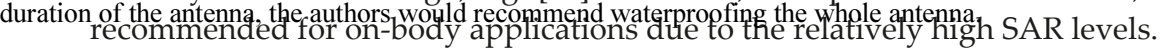

\begin{tabular}{|lccccc|}
\hline & \multicolumn{2}{c}{ Gain (dBi) } & \multicolumn{2}{c|}{ Directivity (dBi) } & \multicolumn{2}{c|}{ Efficiency (\%) } & Sim. \\
\hline & Sim. & Meas. & Sim. & Meas. & 45 \\
\hline Microstrip-fed array (Fig.22a) & 8.6 & 8.0 & 12.1 & 11.9 & 41 \\
\hline Coxial-fed array (Fig.22b) & 11.9 & - & 13.1 & - & 75 \\
\hline
\end{tabular}

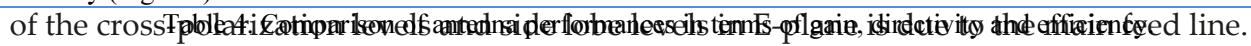

In addition, the gain, directivity and efficiency of these two antennas have been characterized (Table 4). High losses are experienced in the feed line (about $3.3 \mathrm{~dB}$ ). In order to increase the antenna gain and efficiency, the feed line could be shortened or even suppressed (Fig. 22b).

Finally, the antenna performance (i.e. reflection coefficient and radiation) was tested after a number of hand washing cycles. The antenna was measured before and after washing when fully dried; its performance remained unchanged. However, to extend the life duration of the antenna, the authors would recommend waterproofing the whole antenna. 


\begin{tabular}{lcccccc}
\hline & \multicolumn{2}{c}{ Gain (dBi) } & \multicolumn{2}{c}{ Directivity (dBi) } & \multicolumn{2}{c}{ Efficiency (\%) } \\
\hline & Sim. & Meas. & Sim. & Meas. & Sim. & Meas. \\
\hline $\begin{array}{l}\text { Microstrip-fed array (Fig. } \\
\text { 22a) }\end{array}$ & 8.6 & 8.0 & 12.1 & 11.9 & 45 & 41 \\
\hline Coxial-fed array (Fig. 22b) & 11.9 & - & 13.1 & - & 75 & - \\
\hline
\end{tabular}

Table 4. Comparison of antenna performances in terms of gain, directivity and efficiency.

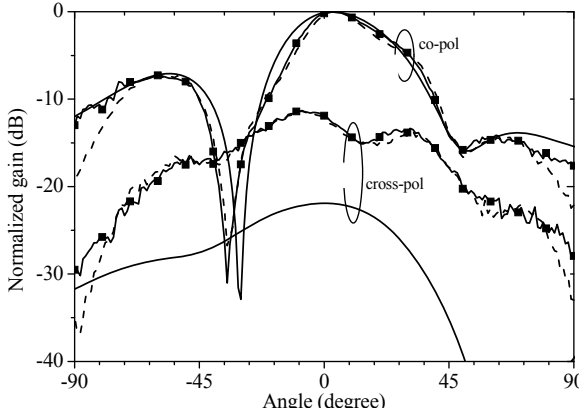

(a) E-plane

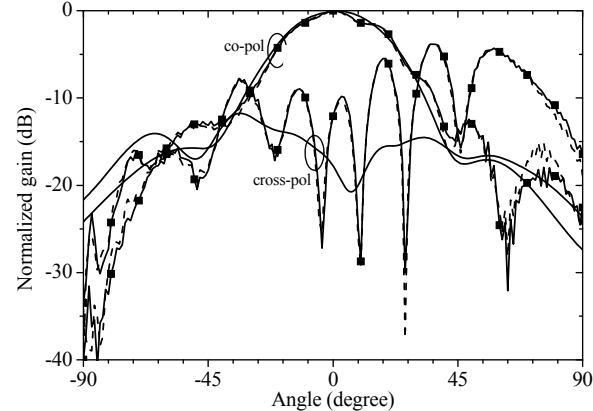

(b) $H$-plane

Fig.25. Normalized radiation patterns in co- and cross-polarization at $60 \mathrm{GHz}$. — Simulation in free space. —- Measurement in free space. - - Measurement on a skin-equivalent phantom.

Figure 25. Normalized radiation patterns in co-and cross-polarization at $60 \mathrm{GHz}$. — Simulation in free space. - - -

Mea3.5.CContinsfiro space. --- Measurement on a skin-equivalent phantom.

Based on computed and measured results, antennas operating at millimeter-waves are very slightly sensitive to the human body. Besides, guidelines regarding the type of antennas, minimizing the interactions with the body, are provided. The feeding of the

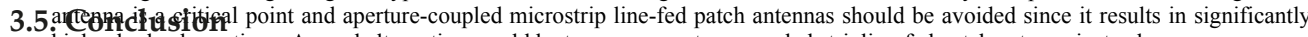
higher body absorptions. A good alternative would be to use an aperture-coupled stripline-fed patch antenna instead.

Finally, textile antennas at millimeter-wave, have been demonstrated with encouraging results. The textile can be accurately

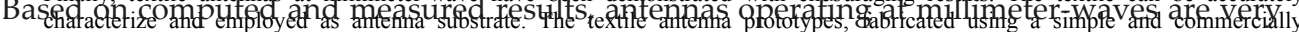

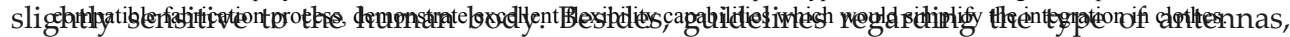

\section{minimizing the interactions with the body are provided. The feeding of the antenna is a critical}

aperture-coupled microstrip line-fed patch antennas should be avoided since it Whereas off-body communications appear to be a good solution at millimeter wayes, on-body communications might be more

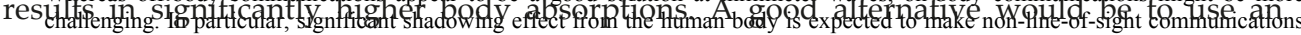

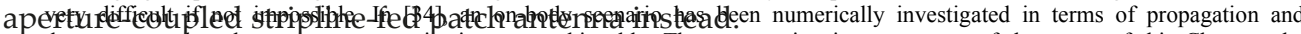
demonstrates that short-range communications are achievable. The propagation issues are out of the scope of this Chapter; the

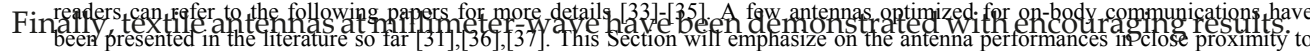
Thehetexdile can be accurately characterize and employed as antenna substrate. The textile antenna prototypes fabricated using a simple and commercially compatible fabrication

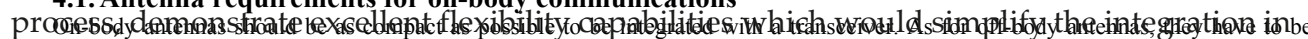
clotight weight and possibly conformable to the human body shape. Because of the high attenuation related to the propagation on a lossy dielectric (i.e. human body), medium-gain antennas $(\sim 12 \mathrm{dBi})$ are required. The radiation pattern should be maximized toward the direction of propagation to minimize losses and make end-fire antennas excellent solutions. As the power is directed toward the body surface, absorptions inside the human is of uppermost concern.

\section{Azqleffirsartopngn-body communications at millimeter waves}

A compact planar and flexible Yagi-Uda antenna covering the 57-64 GHz range designed for on-body communications is presented. The antenna is characterized in free space in terms of reflection coefficient, radiation pattern, and efficiency. The effect of the human

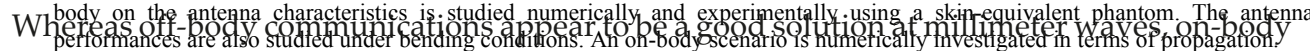
con.munidtemmamighel be more challenging. In particular, significant shadowing effect from High gain antenna is required for a line-of-sight path of human body dimensions. Furthermore, the maximum of the radiation pattern should be tangential to the body surface in order to reduce radiation off the body and thus minimizing interference among different BANs. Hence, a low-profile high-gain antenna with an end-fire radiation pattern printed on a $0.254 \mathrm{~mm}$-thick RT Duroid 5880 substrate $\left(\varepsilon_{r}=2.2, \tan \delta=0.003\right)$ is proposed. The layout is represented in Fig.26. For measurement purpose the antenna prototype is mounted with a V-connector (Fig.27). 
the human body is expected to make non-line-of-sight communications very difficult if not impossible. In [34], an on-body scenario has been numerically investigated in terms of propagation and demonstrates that short-range communications are achievable. The propagation issues are out of the scope of this Chapter; the readers can refer to the following papers for more details [33]-[35]. A few antennas optimized for on-body communications have been presented in the literature so far [31],[36],[37]. This Section will emphasize on the antenna performances in close proximity to the body.

\subsection{Antenna requirements for on-body communications}

On-body antennas should be as compact as possible to be integrated with a transceiver. As for off-body antennas, they have to be light weight and possibly conformable to the human body shape. Because of the high attenuation related to the propagation on a lossy dielectric (i.e. human body), medium-gain antennas $(\sim 12 \mathrm{dBi})$ are required. The radiation pattern should be maximized toward the direction of propagation to minimize losses and make end-fire antennas excellent solutions. As the power is directed toward the body surface, absorptions inside the human is of uppermost concern.

\subsection{End-fire antenna}

A compact planar and flexible Yagi-Uda antenna covering the 57-64 GHz range designed for on-body communications is presented. The antenna is characterized in free space in terms of reflection coefficient, radiation pattern, and efficiency. The effect of the human body on the antenna characteristics is studied numerically and experimentally using a skin-equivalent phantom. The antenna performances are also studied under bending conditions. An on-body scenario is numerically investigated in terms of propagation.

\subsubsection{Antenna model}

High gain antenna is required for a line-of-sight path of human body dimensions. Furthermore, the maximum of the radiation pattern should be tangential to the body surface in order to reduce radiation off the body and thus minimizing interference among different BANs. Hence, a low-profile high-gain antenna with an end-fire radiation pattern printed on a $0.254 \mathrm{~mm}$-thick RT Duroid 5880 substrate $\left(\varepsilon_{r}=2.2, \tan \delta=0.003\right)$ is proposed. The layout is represented in Fig. 26. For measurement purpose the antenna prototype is mounted with a V-connector (Fig. 27).

\subsubsection{Antenna performance in free space}

The reflection coefficient $S_{11}$ of the antenna array is measured with a $110 \mathrm{GHz}$ vector network analyzer (Agilent 8510XF) using a V-connector (Fig. 27). The measured and simulated $S_{11}$ (Fig. 28 ) are below- $10 \mathrm{~dB}$ in the whole $57-64 \mathrm{GHz}$ range. The numerical model does not include the V-connector.

The radiation patterns in $E$-and $H$-planes are plotted in Fig. 29. The simulated and measured radiation patterns at $60 \mathrm{GHz}$ are in good agreement. The cross-polarization remains lower than- $14 \mathrm{~dB}$ in the $E$-and $H$-planes at broadside. The gain was measured by the comparison 
High gain antenna is required for a line-of-sight path of human body dimensions. Furthermore, the maximum of the radiation pattern should be tangential to the body surface in order to reduce radiation off the body and thus minimizing

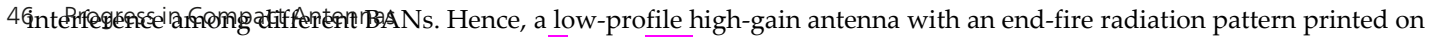
a $0.254 \mathrm{~mm}$-thick RT Duroid 5880 substrate $\left(\varepsilon_{r}=2.2, \tan \delta=0.003\right)$ is proposed. The layout is represented in Fig. 26 . For measurement purpose the antenna prototype is mounted with a V-connector (Fig. 27).

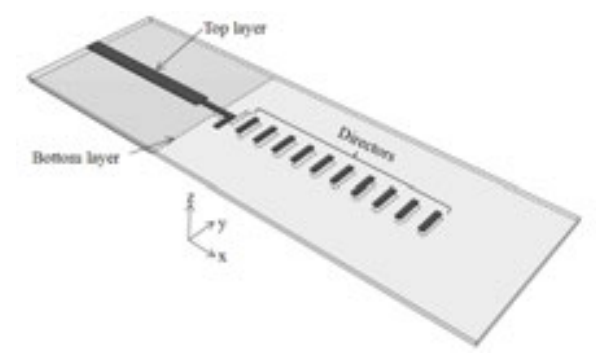

(a)

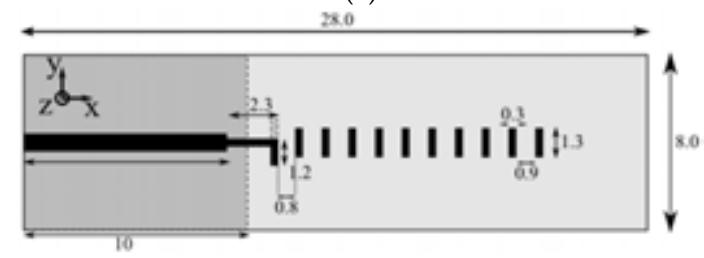

(b)

Figure 26. Layout of the printed Yagi-Uda antenna. Dimensions are in mm. (a) Three dimensions view. (b) Top layer.

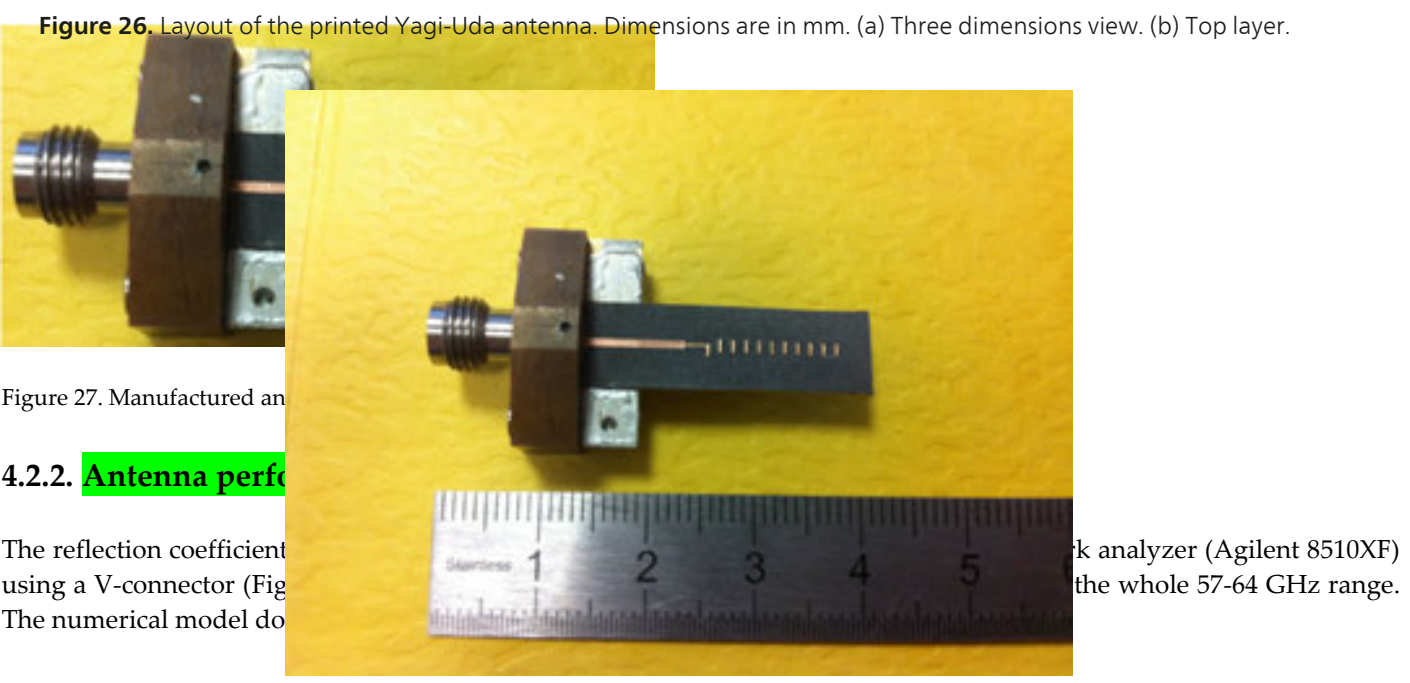

Figure 27. Manufactured antenna with a V-connector.

method with a 20-dBi standard horn. At this frequency, the measured and computed gains equal $11.8 \mathrm{dBi}$ and $12.1 \mathrm{dBi}$ respectively. The losses of the $\mathrm{V}$-connector $(\sim 0.8 \mathrm{~dB})$ at $60 \mathrm{GHz}$ were measured separately and taken out.

The antenna efficiency defined as the measured gain over the computed directivity equals to $86 \%$ at $60 \mathrm{GHz}$. It is in agreement with the simulated efficiency which equals $92 \%$. 
does not include the V-connector.

The radiation patterns in $E$ - and $H$-planes are plotted in Fig.29. The simulated and measured radiation patterns at $60 \mathrm{GHz}$ are in good agreement. The cross-polarization remains lower than $-14 \mathrm{~dB}$ in the $E$ - and $H$-planes at broadside. The gain was measured by the comparison method with a 20-dBi standard horn.At this frequency the measured and computed gains equal 11.8 dBi and 12.1 $\mathrm{dBi}$ respectively. The losses of the $\mathrm{V}$-connector $(\sim 0.8 \mathrm{~dB})$ at $60 \mathrm{GHz}$ were measured separately and taken out 0 (a) $5772 / 58816$ The antenna efficiency defined as the measured gain over the computed directivity equals to $86 \%$ at 60 GHz. It is in agreement with the simulated efficiency which equals $92 \%$.

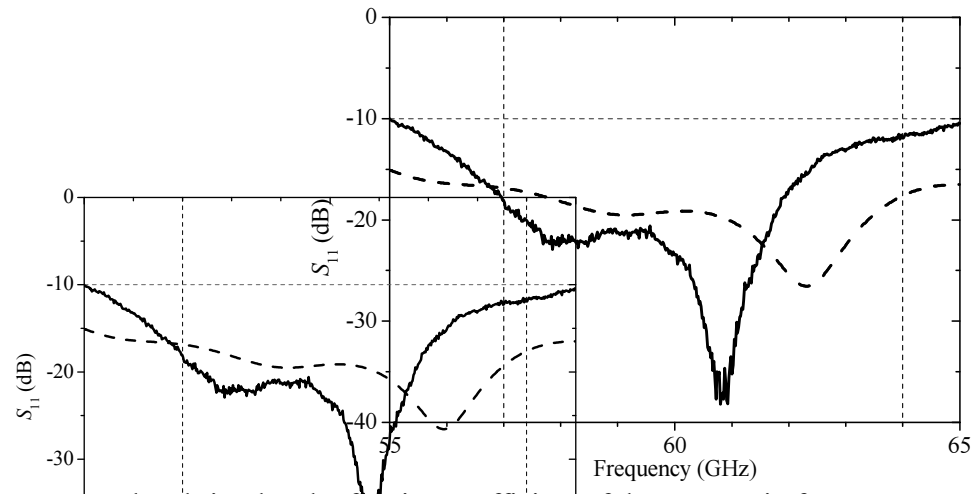

Fig.28. Measured and simulated reflption coefficient of the antenna in free space. — Measurement. — Simulation. Figure 28. Measured and simulated reffection coefficjient of the antenna in free space. --- Measurement. — Simulation.

Figure 28. Measured and simulated reflection coefficient of the antenna in free space. - Measurement. --- Simulation.

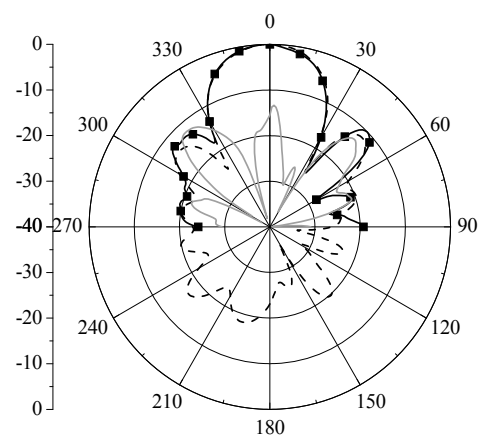

(a)

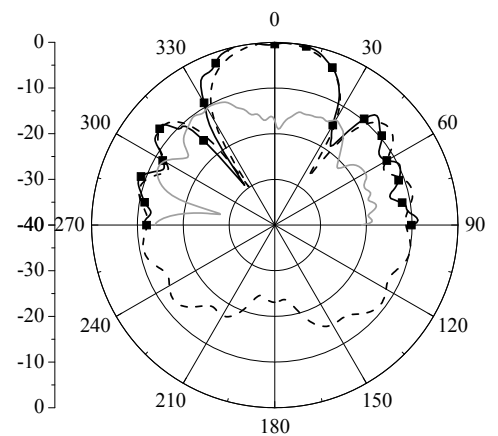

(b)

Figure 29. Measured and simulated radiation patterns in free space at $60 \mathrm{GHz}$ in (a) $E$ - and (b) $H$-planes. - - - Measured co-pol. - - -

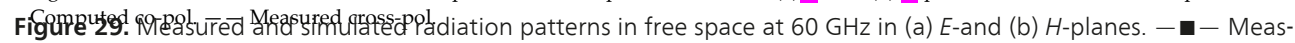
ured co-pol. _ Computed co-pol. _— Measured cross-pol.

\subsubsection{Antenna under bending conditions}

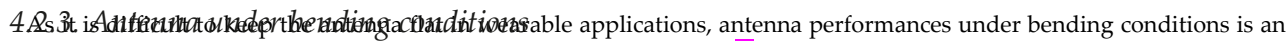
important factor to be examined. The reflection coefficient and the $H$-plane radiation pattern are investigated when the

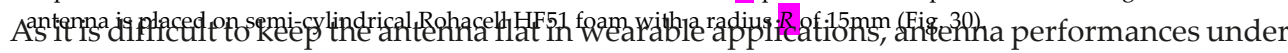

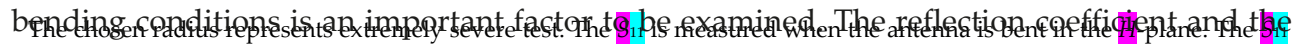

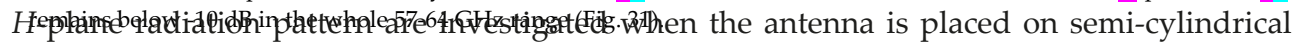
Rohacell $H F 51$ foam with a radius $R$ of $15 \mathrm{~mm}$ (Fig. 30 ). follows the direction of the directors $\left(-46^{\circ}\right)$. Besides, the measured gain equals $11.1 \mathrm{dBi}$. This is in good agreement with

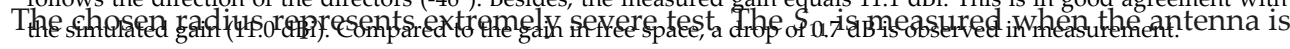
bent in the $H$-plane. The $S_{11}$ remains below-10 dB in the whole 57-64 GHz range (Fig. 31).

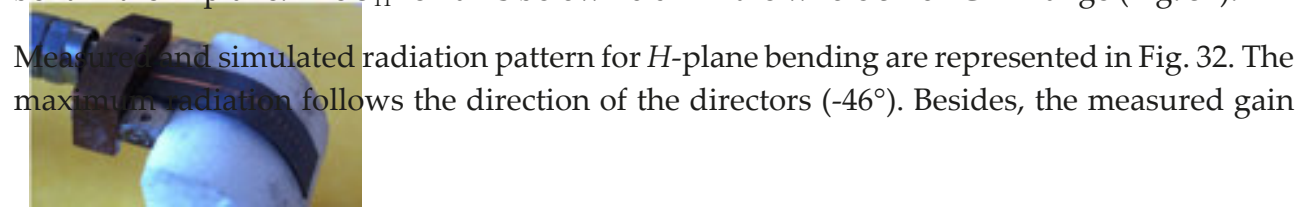

Figure 30. Bending antenna in the $H$-plane placed on a semi-cylindrical foam with $R=15 \mathrm{~mm}$. 

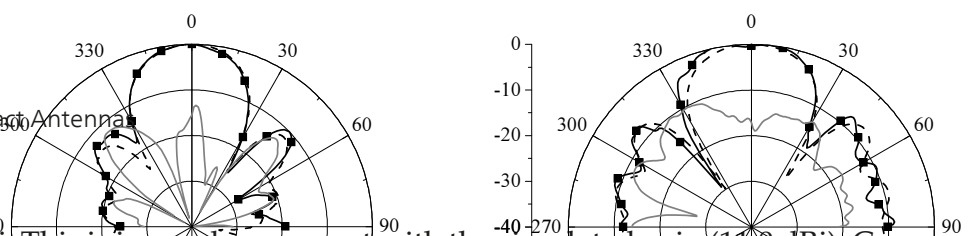

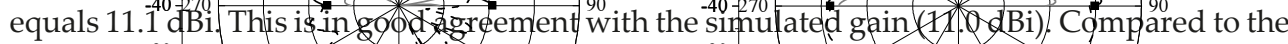
gain in free space, a drop of $Q: Y$. dB is observed in measurement.
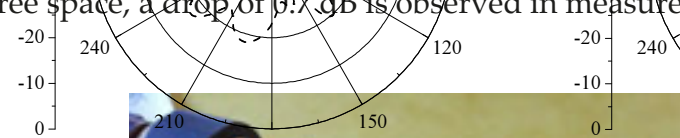

$0]$

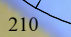

180

(b)

Fig.29. Measured and simulated radiation patterns in free co-pol. — Computed co-poir_- Measured c oss-pol,

$60 \mathrm{GHz}$ in (a) $E$ - and (b) $H$-planes.

Measured

\subsubsection{Antenna under bending conditions}

As it is difficult to keep the antenna flat in wearable applications, antenna performances under bending conditions is an important factor to be examined. The reflection coefficient and the $H$-plane radiation pattern are investigated when the antenna is placed on semi-cylindrical Rohacell HF51 foam with a radius $R$ of $15 \mathrm{~mm}$ (Fig.30).

The chosen radius represents extremely severe test. The $S_{11}$ is measured when the antenna is bent in the $H$-plane. The $S_{11}$ remains below $-10 \mathrm{~dB}$ in the whole 57-64 GHz range (Fig.31).

Measured and simulated radiation pattern for $H$-plane bending are represented in Fig.32. The maximum radiation follows the direction of the directors $\left(-46^{\circ}\right)$. Besides, the measured gain equals $11.1 \mathrm{dBi}$. This is in good agreement with the simulated gain (11.0 $\mathrm{dBi}$ ). Compared to the gain in free space, a drop of $0.7 \mathrm{~dB}$ is observed in measurement.

Figure 30. Bending antenna in the $H$-plane $p$

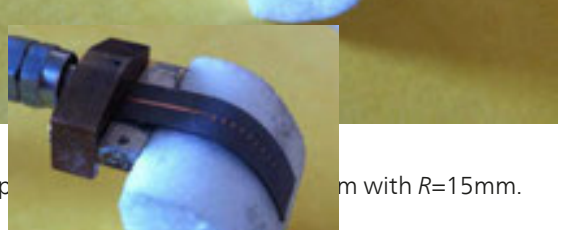

Fig.30. Bending antenna in the $H$-plane placed on a semi-cylindrical foam with $R=15 \mathrm{~mm}$.

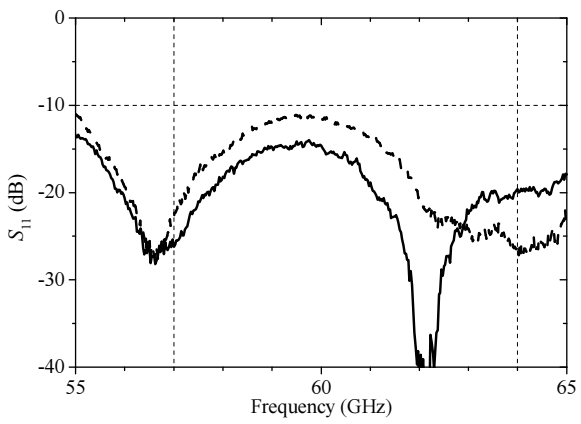

Fig.31. Measured reflection coefficient of the bent antenna mounted on semi-cylindrical foam. - Flat. $-R=15 \mathrm{~mm}$.

Figure 31. Measured reflection coefficient of the bent antenna mounted on semi-cylindrical foam. --- Flat. $R=15 \mathrm{~mm}$.

\subsubsection{Antenna performances on the human body}

The antenna characteristics are assessed when placed on a skin-equivalent phantom (Fig. 33) in terms of reflection coefficient, radiation pattern, gain, and efficiency. The measured reflection coefficients of the antenna mounted on the phantom at different antenna/body spacing $h$ are compared to that obtained in free space in Fig. 34 . For $\mathrm{h}=5 \mathrm{~mm}$, the reflection coefficient is very slightly affected. For $h=2 \mathrm{~mm}$, even though the $S_{11}$ is much more affected and a frequency shift is observed, it remains below-10dB within the whole $57-64 \mathrm{GHz}$. 


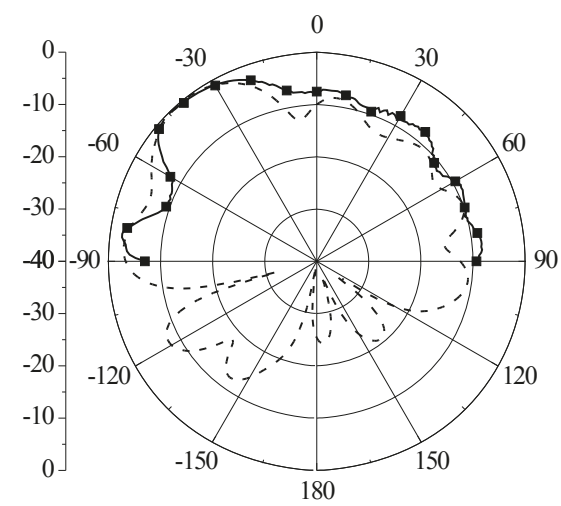

Figure 32. $\mathrm{H}$-plane radiation pattern of the bent antenna $(R=15 \mathrm{~mm})$ mounted on a semi-cylindrical foam. - $\mathbf{-}-$ Measured co-pol. ---- Computed co-pol.

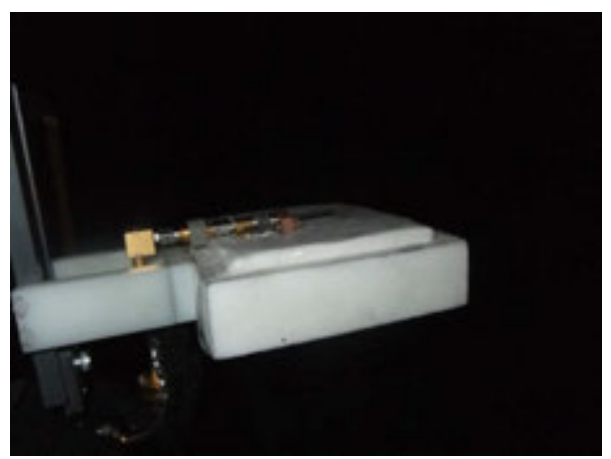

Figure 33. Antenna on the skin-equivalent phantom.

The measured radiation patterns in both $E$-and $H$-planes at $60 \mathrm{GHz}$ of the antenna placed on the skin-equivalent phantom are represented in Fig. 35 for $h=5.6 \mathrm{~mm}$ and $h=2 \mathrm{~mm}$. Both $E$-and $H$-planes are strongly affected by the human body because of reflection on and absorption in the body.

Here, the radiation pattern is titled because of reflections occurring at the air/phantom interface. A tilt of $10^{\circ}$ and $21^{\circ}$ is observed for an antenna/body spacing of $5.6 \mathrm{~mm}$ and $2 \mathrm{~mm}$, respectively. The simulated and measured gains and the simulated efficiency are summarized in Table 5 for different antenna/body spacing. The efficiency decreases with $h$. However, the maximum gain of the antenna increases on the phantom (up to $3 \mathrm{~dB}$ increase for $h=5.6 \mathrm{~mm}$ ). Compared to the free space configuration, radiations toward the human body are significantly reduced because of reflections from and absorptions in the human body. Hence, when the antenna is mounted on the phantom, its performance remains satisfactory in terms of reflection coefficient, radiation pattern, and efficiency. 


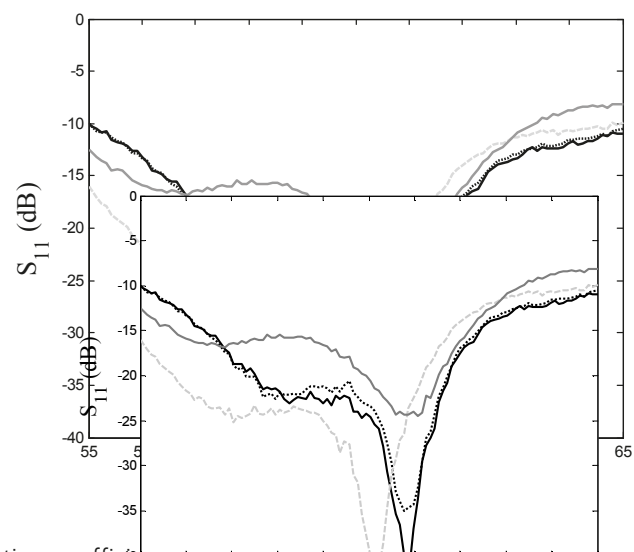

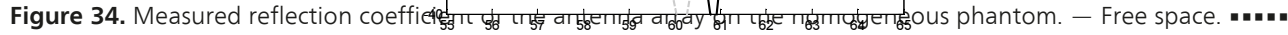
On phantom with $h=5.6 \mathrm{~mm}$.---On phantom with $h=2$ rFirequenQn(Ghantom with $h=0 \mathrm{~mm}$.

Fig.34. Measured reflection coefficient of the antenna array on the homogeneous phantom.- Free space. ....On phantom with $h=5.6 \mathrm{~mm}$. - - On phantom with $h=2 \mathrm{~mm}$. - On phantom with $h=0 \mathrm{~mm}$.

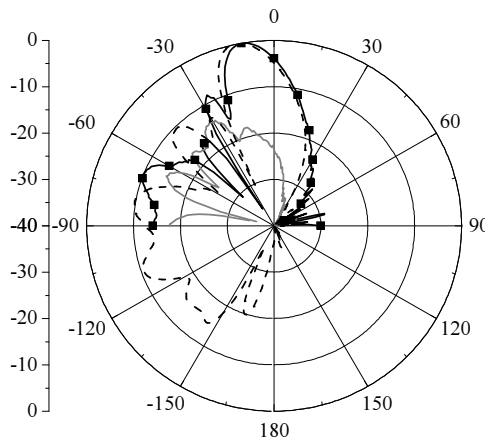

(a) E-plane, $h=5.6 \mathrm{~mm}$

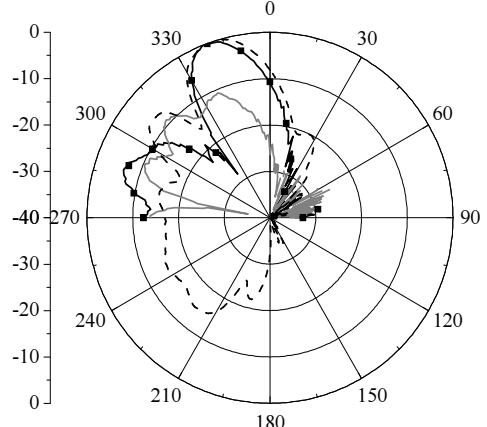

(d) E-plane, $h=2 \mathrm{~mm}$

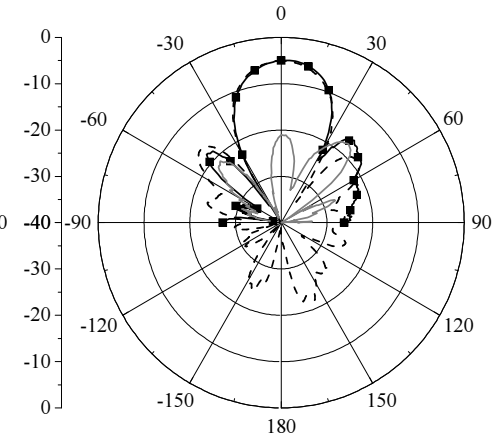

(b) H-plane, $h=5.6 \mathrm{~mm}$

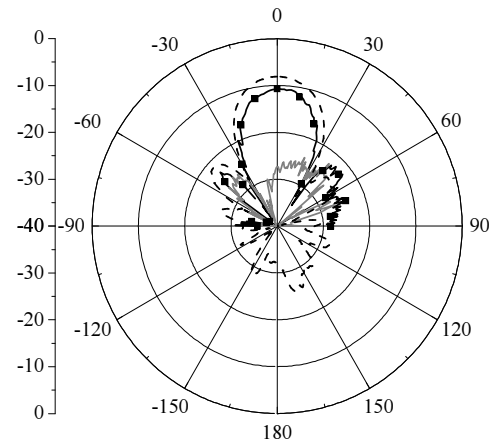

(e) H-plane, $h=2 \mathrm{~mm}$

Fig.35. Measured and simulated radiation patterns on the skin-equivalent phantom at $60 \mathrm{GHz}$. —- Measured co-pol.

Figure 35. Measured and simulated radiation patterns on the skin-equivalent phantom at $60 \mathrm{GHz}$. - - - Measured

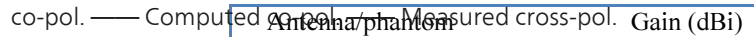

\begin{tabular}{|c|c|c|c|}
\hline \multicolumn{3}{|l|}{ hepha/pl } & \multirow{2}{*}{$\begin{array}{c}\text { Simulated } \\
\text { efficiency }(\%)\end{array}$} \\
\hline separation $h(\mathrm{~mm})$ & Simulated & Measured & \\
\hline$\infty$ & 12.1 & 11.8 & 92.2 \\
\hline 5.6 & 15.1 & 15.2 & 74.9 \\
\hline 2 & 13.6 & 13.6 & 68.2 \\
\hline
\end{tabular}

Table 5. Antenna gain and efficiency for different antenna/body spacing. 


\begin{tabular}{|c|c|c|c|}
\hline \multirow{2}{*}{$\begin{array}{l}\text { Antenna/phantom separation } \mathrm{h} \\
\qquad(\mathrm{mm})\end{array}$} & \multicolumn{2}{|c|}{ Gain (dBi) } & \multirow{2}{*}{-Simulated efficiency (\%) } \\
\hline & Simulated & Measured & \\
\hline$\infty$ & 12.1 & 11.8 & 92.2 \\
\hline 5.6 & 15.1 & 15.2 & 74.9 \\
\hline 2 & 13.6 & 13.6 & 68.2 \\
\hline
\end{tabular}

Table 5. Antenna gain and efficiency for different antenna/body spacing.

\subsection{Conclusions}

A compact planar Yagi-Uda antenna covering the 57-64 GHz range has been designed for onbody communications. The effect of the human body on the antenna characteristics has been studied numerically and experimentally using a skin-equivalent phantom. It was shown that the distance between the antenna and the human body has a strong impact on the antenna performances. The antenna was also studied under bending conditions demonstrating satisfactory performances. The same antenna has been successfully optimized and fabricated on textile [31].

\section{Conclusion}

Challenges and progress in antennas and their interaction with the human body in bodycentric scenarios at millimeter-wave frequencies have been presented in this Chapter. Recent progress in manufacturing and modeling experimental phantoms has been discussed. These phantoms play a key role in characterizing the antenna performance in close proximity to the human body.

As far as off-body communications are concerned, it was shown that the feeding type is an important factor since it can strongly influence absorption in the human body. In addition, performances of patch antenna arrays in close proximity to the human body have been evaluated showing very slight impact on the antenna performance. Besides, a textile patch antenna array, operating at millimeter waves, was successfully demonstrated using a commercially-available textile. An accurate and low-cost fabrication process has been introduced. Research work should now be focused on the interconnections between textile antennas and Radio Frequency Integrated Circuits (RFIC) since this issue has not been tackled yet.

Finally, as end-fire antennas appear to be the best solution for on-body communications, a Yagi-Uda antenna has been investigated. It appears that the antenna radiation pattern is strongly affected by the separation between the antenna and the human body. This antenna is robust against bending which is an important asset if this antenna would be implemented on textile as shown in [31]. Other antenna designs for on-body communications were introduced in [38].

While these results are promising, millimeter-wave wireless systems still have considerable challenges to overcome to enable mass commercialization. First, mm-wave wireless must 
address challenging RF impairments such as fast fading and delay spread conditions making demodulation and equalization particularly difficult with reasonable architectures and complexities. Second, millimeter-wave transceivers require giga-samples per second (GS/s) scale data-converters with considerable resolutions leading to high power consumption (even in advanced technology nodes). Finally, mm-wave schemes must prove themselves competitive with advanced and adaptive modulation and channel coding schemes (256 QAM and beyond) like 802.11 ac 5 th generation WiFi that can also reach high data rates $(6.77 \mathrm{Gbit} / \mathrm{s}$ nominal) while being built upon existing wireless hardware and infrastructure in the $5.83 \mathrm{GHz}$ ISM band.

\section{Acknowledgements}

This work was supported by French National Research Agency (ANR) under Grant ANR-09RPDOC-003-01 (Bio-CEM project), by Labex CominLabs (ANR program "Investing for the Future" ANR-10-LABX-07-01) and Brittany Region under ResCor/BoWi project and by National Center for Scientific Research (CNRS), France. Part of this work was performed using HPC resources from GENCI-IDRIS (grant 2013-050779).

\section{Author details}

Nacer Chahat ${ }^{1}$, Maxim Zhadobov ${ }^{2}$ and Ronan Sauleau ${ }^{2}$

1 NASA Jet Propulsion Laboratory/California Institute of Technology, Pasadena, CA, USA

2 University of Rennes 1, Rennes, France

\section{References}

[1] P. S. Hall and Y. Hao, "Antennas and propagation for body centric communications systems," Artech House, Norwood, MA, 2006, ISBN-10: 1-58053-493-7.

[2] D. Guha and Y. M. M. Antar, "Microstrip and printed antennas: new trends, techniques and applications," Wiley-Blackwell, 2011, ISBN-10: 0470681926.

[3] T. Baykas, C. S. Sum, Z. Lan, J. Wang, M. A. Rahman, and H. Harada, "IEEE 802.15.3c: the first IEEE wireless standard for data rates over $1 \mathrm{~Gb} / \mathrm{s}$," IEEE Communications Mag., vol. 49, no. 7, pp. 114-121, Jul. 2011.

[4] S. L. Cotton, W. G. Scanlon, and P. S. Hall, "A simulated study of co-channel interBAN interference at $2.45 \mathrm{GHz}$ and $60 \mathrm{GHz}$," Europ. Wirel. Techn. Conf., Paris, France, pp. 61-64, Sep. 2010. 
[5] M. Kojima, et al., "Acute ocular injuries caused by 60-Ghz millimeterwave exposure," Health Phys., vol. 97, no. 3, pp. 212-218, Sept. 2009.

[6] H. A. Kues, S. A. D'Anna, R. Osiander, W. R. Green, and J. C. Monahan, “Absence of ocular effects after either single or repeated exposure to $10 \mathrm{~mW} / \mathrm{cm} 2$ from a $60 \mathrm{GHz}$ CW source," Bioelectromagnetics, vol. 20, no. 8, pp. 463-473, Dec. 1999.

[7] F. A. Duck, "Physical Properties of Tissue: a comprehensive reference network," U.K.: Academic, ISBN 0122228006, 1990.

[8] O. P. Gandhi and A. Riazi, "Absorption of millimeter waves by human beings and its biological implications," IEEE Trans. Microwave. Theory Tech., vol. 34, no. 2, pp. 228 235, Feb. 1986.

[9] C. M. Alabaster, "Permittivity of human skin in millimetre wave band," Elec. Lett., vol. 39, no. 21, pp. 1521-1522, Oct. 2003.

[10] S. Gabriel, R. W. Lau, and C. Gabriel, "The dielectric properties of biological tissues: III. Parametric models for the dielectric spectrum of tissues," Phys. Med. Biol., vol. 41, no. 11, pp. 2271-2293, Nov. 1996.

[11] S. I. Alekseev and M. C. Ziskin, "Human skin permittivity determined by millimeter wave reflection measurements," Bioelectromagnetics, vol. 28, no. 5, pp. 331-339, Jul. 2007.

[12] N. Chahat, M. Zhadobov, and R. Sauleau, "Broadband tissue-equivalent phantom for BAN applications at millimeter waves," IEEE Transactions on Microwave Theory and Techniques, vol. 60, no. 7, pp. 2259-2266, Jul. 2012.

[13] N. Chahat, M. Zhadobov, R. Sauleau, and S. Alekseev, "New method for determining dielectric properties of skin and phantoms at millimeter waves based on heating kinetics," IEEE Transactions on Microwave Theory and Techniques, vol. 60, no. 3, pp. 827-832, March 2011.

[14] P. F. M. Smulders, "Impact of regulations on feasible distance between $60 \mathrm{GHz}$ devices," Europ. Conf. Antennas Propag., EuCAP'2010, Barcelona, Spain, Apr. 12-16, 2010.

[15] S. L. Cotton, W. G. Scanlon, and B. K. Madahar, "Millimeter-wave soldier-to-soldier communications for covert battlefield operations," IEEE Communications Mag., vol. 47, no. 10, pp. 72-81, Oct. 2009.

[16] P. F. M. Smulders, "Statistical characterization of $60-\mathrm{GHz}$ indoor radio channels," IEEE Trans. Antennas Propag., vol. 57, no. 10, pp. 2820-2829, Oct. 2009.

[17] R. C. Daniels, J. N. Murdock, T. S. Rappaport, and R. W. Heath, “60 GHz wireless: up close and personal," IEEE Microwave Magazine, vol. 11, no. 7, pp. 44-50, Dec. 2010.

[18] S. Collonge, G. Zaharia, and G. El Zein, "Wideband and dynamic characterization of the $60 \mathrm{GHz}$ indoor radio propagation - future home WLAN architectures," Ann. Telecommun., vol. 58, no. 3-4, Mar.-Apr. 2003. 
[19] K. L. Wong and C. I. Lin, “Characteristics of a 2.4-GHz compact shorted patch antenna in close proximity to a lossy medium," Microw. Opt. Technol. Lett., vol. 45, no. 6, pp. 480-483, Jun. 2005.

[20] L. Vallozzi, W. Vandendriessche, L. Vallozzi, W. Vandendriessche, H. Rogier, C. Hertleer, and M. L. Scarpello, "Wearable textile GPS antenna for integration in protective garments," Europ. Conf. Antennas Propag., EuCAP'2010, Barcelona, Spain, Apr. 12-16, 2010.

[21] M. M. Khan, A. Alomainy, and Y. Hao, “Dual band and diverse radiation pattern antenna for power efficient and reliable on-body and off-body communications for healthcare applications," IEEE Ant. Propag. Society Int. Symp., Spokane (WA), Jul. 2011.

[22] "Guidelines for limiting exposure to time-varying electric, magnetic, and electromagnetic fields (up to $300 \mathrm{GHz}$ )," Health Phys., vol. 74, no. 4, pp. 494-522, 1998.

[23] M. F. Karim, Y.-X. Guo, M. Sun, J. Brinkhoff, L. C. Ong, K. Kang, and F. Lin, “Integration of SiP-Based 60-GHz $4 \times 4$ Antenna Array With CMOS OOK Transmitter and LNA," IEEE Trans. Microw. Theory Tech., vol. 59, no. 7, pp. 1869-1878, Jul. 2011.

[24] N. Chahat, M. Zhadobov, L. Le Coq, S. Alekseev, and R. Sauleau, "Characterization of the Interactions between a 60-GHz Antenna and the Human Body in an Off-Body Scenario," IEEE Transactions on Antennas and Propagation, vol. 60, no. 12, pp. 59585965, Dec. 2012.

[25] P. Coquet, R. Sauleau, K. Shinohara, and T. Matsui, "Multi-layer microstrip antennas on quartz substrates. Technological considerations and performance at $60 \mathrm{GHz}$," Microw. Opt. Technol. Lett., vol. 40, no. 1, pp. 41-47, Jan. 2004.

[26] B. Biglarbegian, M. Fakharzadeh, D. Busuioc, M. R. Nezhad-Ahmadi, and S. SafaviNaeini, “Optimized microstrip antenna arrays for emerging millimeter-wave wireless applications," IEEE Trans. Antennas Propag., vol. 59, no. 5, pp. 1742-1747, May 2011.

[27] B. Chantraine-Bares, R. Sauleau, L. Le Coq, and K. Mahdjoubi, "A new accurate design method for millimeter-wave homogeneous dielectric substrate lens antennas of arbitrary shape," IEEE Trans. Antennas Propag., vol. 53, no. 3, pp. 1069-1082, March 2005.

[28] M. Zhadobov, N. Chahat, R. Sauleau, C. Le Quement, Y. Le Dréan, “Millimeter-wave interactions with the human body : state of knowledge and recent advances," International Journal of Microwave and Wireless Technologies, vol. 3, no. 2, pp. 237-247, April 2011.

[29] N. Chahat, M. Zhadobov, and R. Sauleau, "60-GHz textile antenna array for bodycentric communications," IEEE Transactions on Antennas and Propagation, vol. 61, no. 4, pp. 1816-1824, Apr. 2013. 
[30] S. Hage-Ali, N. Tiercelin, P. Coquet, R. Sauleau, H. Fujita, V. Preobrazhensky, and P. Pernod, "A millimeter-wave microstrip antenna array on ultra-flexible micromachined polydimethylsiloxane (PDMS) polymer," IEEE Antennas Wirel. Propag. Lett., vol. 8, pp. 1306-1309, 2009.

[31] N. Chahat, M. Zhadobov, and R. Sauleau, "Wearable end-fire textile antenna for onbody communications at $60 \mathrm{GHz}$," IEEE Antennas Wirel. Propag. Letters, vol. 11, pp. 799-802, 2012.

[32] I. Locher, M. Klemm, T. Kirstein, and G. Troster, "Design and characterization of purely textile patch antennas," IEEE Trans. Advanced Packaging, vol. 29, no. 4, pp.777788, Nov. 2006.

[33] T. Mavridis, L. Petrillo, J. Sarrazin, D. Lautru, A. Benlarbi-Delai, and P. De Doncker, "Theoretical and Experimental Investigation of a $60 \mathrm{GHz}$ Off-Body Propagation Model," IEEE Transactions on Antennas and Propagation, in press.

[34] N. Chahat, G. Valerio, M. Zhadobov, and R. Sauleau, “On-body propagation at 60 GHz," IEEE Transactions on Antennas and Propagation, vol. 61, no. 4, pp. 1876-1888, Apr. 2013.

[35] A. Guraliuc, M. Zhadobov, V. Guido, N. Chahat, and R. Sauleau, "Effect of Textile on the Propagation Along the Body at $60 \mathrm{GHz}, "$ IEEE Transactions on Antennas and Propagation, in press.

[36] X. Y. Wu and P. S. Hall, "Substrate integrated wavegide Yagi-Uda antenna," Electron. Lett., vol. 11, no. 23, pp. 1541-1542, Nov. 2010.

[37] X. Y. Wu, L. Akhoondzadeh-Asl, and P. S. Hall, "Printed Yagi-Uda array for on-body communication channels at $60 \mathrm{GHz}, "$ Microw. Opt. Technol. Lett., vol. 53, no. 12, pp. 2728-2730, Dec. 2011.

[38] A. Pellegrini, A. Brizzi, L. Zhang, K. Ali, Y. Hao, X. Wu, C. C. Constantinou, Y. Nechayev, P. S. Hall, N. Chahat, M. Zhadobov, and R. Sauleau, “Antennas and propagation for body centric wireless communications at millimeter wave frequencies: a review," IEEE Antennas and Propagation Magazine, vol. 55, no. 4, Nov. 2013. 
